\title{
Streak instability and generation of hairpin-vortices by a slotted jet in channel crossflow: experiments and linear stability analysis
}

\author{
Jimmy Philip, 1, 2, a) Michael Karp, 2, b) and Jacob Cohen ${ }^{2, c)}$ \\ 1) Department of Mechanical Engineering, University of Melbourne, VIC 3010, \\ Australia \\ ${ }^{2)}$ Faculty of Aerospace Engineering, Technion - Israel Institute of Technology, \\ Haifa 32000, Israel
}

(Dated: 22 December 2015)

\begin{abstract}
Streaks and hairpin-vortices are experimentally generated in a laminar plane Poiseuille crossflow by injecting a continuous jet through a streamwise slot normal to the crossflow, with air as the working media. Small disturbances form stable streaks, however, higher disturbances cause the formation of streaks which undergo instability leading to the generation of hairpin vortices. Particular emphasis is placed on the flow conditions close to the generation of hairpin-vortices. Measurements are carried out in the cases of natural and phase-locked disturbance employing smoke visualisation, PIV and hot-wire anemometry, which include, the dominant frequency, wavelength and the disturbance shape (or eigenfunctions) associated with the coherent part of the velocity field. A linear stability analysis for both one- and two-dimensional base-flow is carried out to understand the mechanism of instability and good agreement of wavelength and eigenfunctions are obtained when compared to the experimental data, and a slight under-prediction of the growth-rates by the linear stability analysis consistent with the final nonlinear stages in transitional flows. Furthermore, an energy analysis for both the temporal and spatial stability analysis revels the dominance of the symmetric varicose mode, again, in agreement with the experiments, which is found to be governed by the balance of the wallnormal shear and dissipative effects rather than the spanwise shear. In all cases the anti-symmetric sinuous modes governed by the spanwise shear are found to be damped both in analysis and in our experiments.
\end{abstract}

PACS numbers: Valid PACS appear here

Keywords: Suggested keywords

\section{INTRODUCTION}

The present paper aims to present experimental data on the formation of coherent structures when the fluid is injected through an almost 'streamwise independent' slot into a channel crossflow, for low jet to crossflow ratios. The experiments are specifically tuned to understand the instability of streaks leading to the formation of hairpin-vortices. The experimental data is supplemented and compared with the linear stability theory based on the Orr-Sommerfeld equation for one-dimensional and employing Floquet theory for two-dimensional base-flows.

Since the observation of streaks and hairpin vortices in turbulent boundary layers ${ }^{1}$, these coherent structures have come under intense investigations regarding their characterisation, generation and sustenance mechanisms ${ }^{2,3}$. The presence of streaky structures also in transitional flows ${ }^{4}$ and their further breakup and appearance of hairpin-vortices have led to the

\footnotetext{
a) Electronic mail: jimmyp@unimelb.edu.au

b) Electronic mail: mkarp@technion.ac.il

c) Electronic mail: aerycyc@gmail.com
} 
universality of these two coherent structures being regarded as basic elements in transitional and turbulent wall-bounded shear flows. Furthermore, coherent structures (including streaks and hairpin-vortices) are an integral part of the jet-in-crossflow scenarios ${ }^{5-7}$. In the last few years there has been an increased amount of research in this area ${ }^{8-11}$. Interestingly, most of the jet-in-crossflow studies have primarily dealt with jet issuing with a circular cross-section, whereas technological applications are not limited to this specific geometry. In this regard, the current investigation is related to both the formation of coherent structures in transitional/turbulent flows, as well as, provides a different geometric configuration for understanding jets in crossflows.

For the case of coherent structures in turbulent/transitional flows, the investigations have progressed both experimentally and numerically in two different but complementary directions. First, the study of coherent structures in the 'real' turbulent/transitional flows, and second, the investigation of structures in 'quiet' laminar base-flows by following artificially generated disturbances. The former has the advantage of being the 'real' flow but the process of investigation is complicated by the noise that is inherent in any turbulent flow and by the complexity of extraction of the embedded coherent structures. The later is more suitable to isolate the major effects associated with the coherent structures and is thus effective to study their formation, growth and decay/breakdown, however, lacks the high shear stress that is associated with the turbulent flows. We pursue the second method of investigation by following artificially generated streaks (resulting from a continuous injection of air through a narrow slot at the bottom of a channel) and their further breakdown into hairpin-vortices ${ }^{12-14}$.

The origin of streaks in transitional flows is attributed to the transient growth process ${ }^{15-18}$. There have also been several attempts to employ the same process, within the realm of linearised Navier-Stokes equations, in order to explain the origin of streaks in turbulent flows ${ }^{19,20}$. Furthermore, there are other proposals that also exist for the explanation of streaks in turbulent flows, for example, considering streaks as the flow between two combined stretched legs of a passing array of hairpin-vortices ${ }^{21}$. For the origin of hairpins in turbulent flow, the major proposed directions are: the generation of many hairpin-vortices from a single primary one ${ }^{22,23}$; strong localised disturbances giving rise to hairpin-vortices in the presence of a background shear flow $^{24-26}$; and the generation of hairpin-vortices due to the instability of streaky base-flow profile ${ }^{3,27-29}$. In transitional flows the instability of streaks is by far the most studied mechanism that leads to the explanation of the array of hairpin-vortices that have been observed. The study of streak instability has dominated the investigation of transition process in boundary layers ${ }^{17,30}$, pipe ${ }^{31-33}$ and channel flows ${ }^{13,34}$. Furthermore, the non-linear generation of the packet of hairpins has been reproduced by a minimal element mode ${ }^{35}$ consisting of three elements: simple shear as a base-flow, a counter-rotating vortex pair having finite streamwise vorticity magnitude and a two-dimensional wavy (in the streamwise direction) spanwise vortex sheet, along with verification by experiments both in pipe and channel flows.

For the case of jet issuing from a streamwise slot, which has the streamwise to spanwise aspect ratio $A \gg O(1)$, it is known ${ }^{12,13,36}$ that three different types of coherent motions are observed as the jet to crossflow velocity ratio $v_{0}$ is increased. Starting with extremely low $v_{0}(\approx 0)$, initially a single leg of streaky structure is seen and with increasing $v_{0}$ a double legged structure (like in figure 3a) is observed, which when increased beyond a threshold (depending upon the crossflow Reynolds number) ${ }^{13}$ leads to the shedding of hairpin-vortices. This scenario is slightly different for the case $A \approx O(1)$, such as in the case of jet issuing from a circular hole, where a plethora of coherent motions are observed ${ }^{5}$. The most prominent coherent structure is the stationary horse-shoe vortex for $A \approx O(1)$, which is absent for $A \gg O(1)$ for the range of $v_{0}$ investigated.

In the present investigation with $A \gg O(1)$, we focus on relatively low values of $v_{0}$ where the streaks are produced and their instability is imminent, which will lead to a breakdown and subsequent generation of hairpin-vortices. The rest of the paper is arranged in the following manner. The next section II describes the experimental setup and measurement techniques focusing on phase-locking the data by imposing a very small perturbation on the 


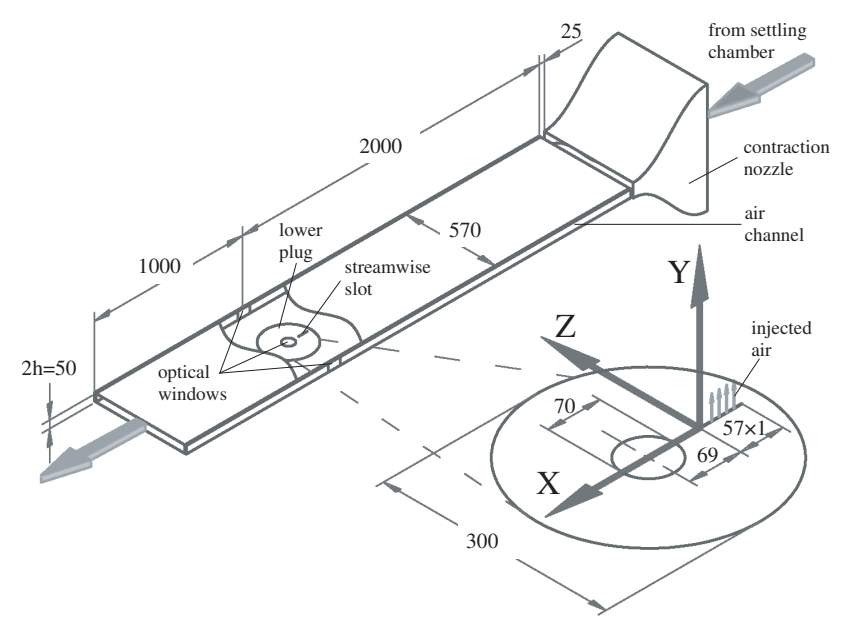

FIG. 1. Air channel facility. All dimensions in millimetres.

jet. Section III presents the experimental results for both 'natural' and phase-locked cases. Subsequently, the linear stability analysis is carried out in section IV for the cases of 1D base-flow (wallnormal variation) and 2D base-flow (wallnormal and spanwise variations), and comparisons with the experiments are made where it is possible. Finally, we close with summary and conclusions in section V.

\section{EXPERIMENTAL SETUP}

The main experimental setup is the same which has been employed previously ${ }^{12,13}$ with additions to carry out phase locked data measurements. The channel flow is an open circuit air facility consisting of a centrifugal blower, a noise reduction chamber, a diffuser and a settling chamber followed by a contraction nozzle and a $3 \mathrm{~m}$ long glass channel that is placed horizontally downstream of the contracting nozzle. As sketched in figure 1 , the channel consists of two glass plates separated by $50 \mathrm{~mm}(2 h)$ bars, positioned to give a channel width of $570 \mathrm{~mm}$. Disturbances are introduced into the laminar Poiseuille flow by continuous air injection through a streamwise slot of size $57 \times 1 \mathrm{~mm}$, drilled in the lower plug. The (normalised) coordinate system has the $X-, Y$ - and $Z$ - axes aligned in the streamwise, wall-normal and spanwise directions, respectively. The origin is at the downstream edge of the injection slot as shown in figure 1 . The flow quantities are normalised with the channel centreline velocity $U_{c l}$ and the half-channel height $h=25 \mathrm{~mm}$, for example, $X=x / h$.

Velocity measurements are accomplished via hot-wire anemometry and Particle Image Velocimetry (PIV), accompanied by flow visualisations using the PIV setup, however, with different seeding conditions. The general PIV and hot-wire setup used in this study are shown in figure 2. The continuous disturbance jet flow is generated using a 'low pressure air', which passes through a metering valve, a mixing tank, and finally injected into the channel flow facility through the streamwise independent slot.

For PIV and flow visualisation two mutually perpendicular laser sheets are formed independently, the vertical $X-Y$ plane, and the horizontal $X-Z$ plane. For most part, the laser system for PIV is a 'Big-Sky' laser with $150 \mathrm{~mJ} /$ pulse at $15 \mathrm{~Hz}$, whereas for flow visualisation a 'Spectra-Physics' laser with $400 \mathrm{~mJ} /$ pulse at $2 \mathrm{~Hz}$ is also used. For flow visualisation, tracer particles are added to the disturbance flow only (not the main channel flow), produced by a smoke machine via the condensation of oil vapour. For the PIV measurements, on the other hand, the whole channel is also seeded with olive oil particles much ahead of the channel inlet. Thus, for the measurements of disturbance with the base-flow, two seeding sources are used in tandem. For PIV, a Redlake camera with $1000 \times 1000$ pixels 


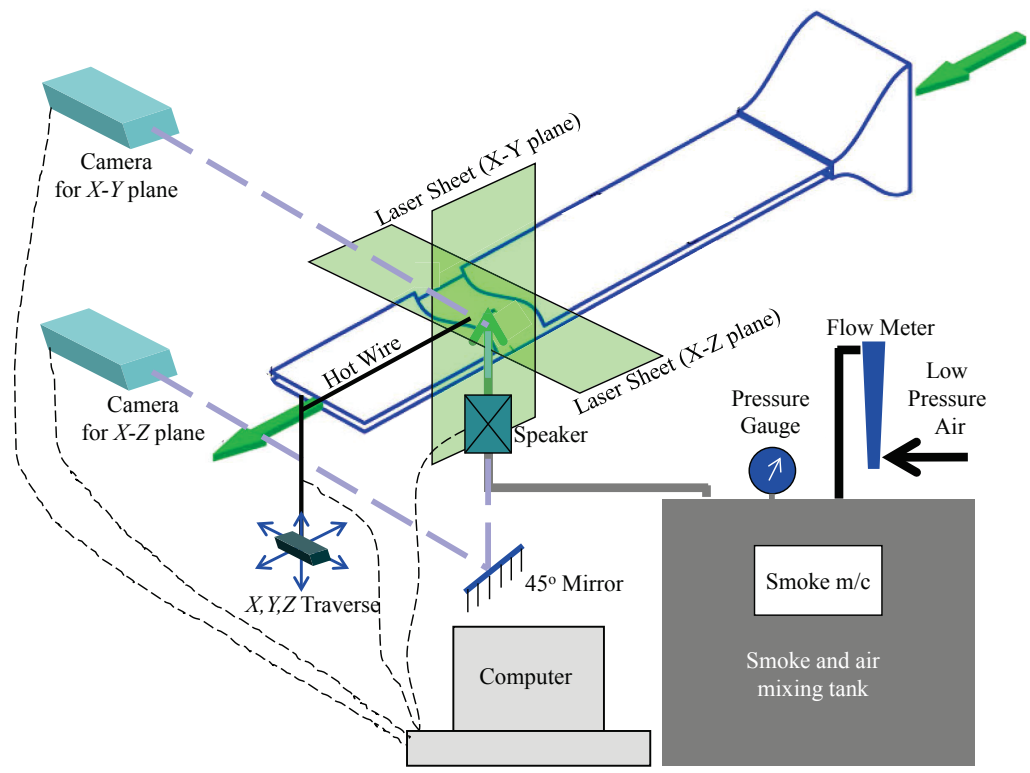

FIG. 2. (Colour online) Schematic of the air channel facility, showing the hot wire measurement system as well as the secondary flow setup for disturbance generation and the PIV setup capable of phase-locked velocity measurements in the $X-Y$ and $X-Z$ planes, controlled and synchronised by the computer.

is used. The particle images are processed with the PIV software INSIGHT ${ }^{\mathrm{TM}}$ (by TSI Inc.).

For velocity measurements using hot-wire anemometry, two separate hot wires are used: one fixed, which continuously measures the centerline velocity, and a second one which is introduced from the exit of the channel and mounted on a traversing mechanism capable of movements in $X, Y$ and $Z$ directions. Both the hot wires are $5 \mu \mathrm{m}$ tungsten wires welded to standard Dantec 55P11 prongs with a length of $1.25 \mathrm{~mm}$ (which is the length over which the velocity is averaged), and calibrated with the help of a pitot tube, placed close to the exit of the channel. Notice that above the slotted jet the velocity is about $30 \%$ of the centreline velocity, which will cause measurement errors if the hot-wire is placed in the usual way with the wires parallel to $z$-direction. Therefore, the main movable hot-wire is mounted with wire in the wall-normal $y$-direction. Using the cooling law: $u_{e f f}^{2}=u_{\perp}^{2}+k^{2} u_{\|}^{2}$ (with the usual value of $k=0.2$ ) and with $u_{\|}$of $30 \%$ will produce an error of only $0.18 \%$, which is negligible.

For phase-locked measurements, a box with two audio speakers is placed immediately before the disturbance fluid enters the channel (see figure 2). The speakers produce a periodic disturbance with a desired frequency which can be phase locked with the PIV/hotwire measurements. With hot-wire, the measurements are continuously sampled along with the speaker input signal, whereas for the PIV a separate program is written in LabView ${ }^{\mathrm{TM}}$, which can trigger the laser at a specified phase shift with respect to the input signal of the speaker recoded at $1 \mathrm{~Hz}$. Note that for the phase-locked measurements the recording rate is not particularly relevant.

The various flow parameters are, the amount of disturbance injected into the channel, the crossflow velocity and the forcing frequency of the injected disturbance. The normalised disturbance amplitude is defined as the average injection velocity normalised by the channel crossflow centerline velocity: $v_{0}=Q_{i n j} /\left(S_{i n j} U_{c l}\right)$, where $Q_{i n j}$ is the injection flow rate and $S_{i n j}$ is the slot cross-section area. In the present work, the forcing frequency (corresponding closely to the natural shedding frequency of hairpin-vortices) has been used, with a very small amplitude for the mere purpose of phase locking the velocity measurements. The base- 


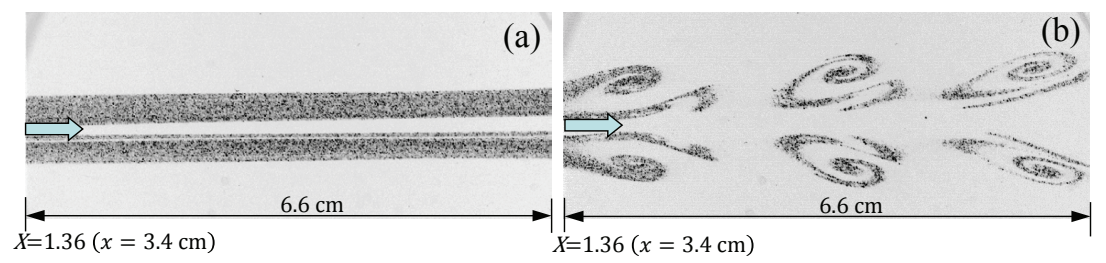

FIG. 3. Smoke flow visualisation of disturbance flow for $R e=1660$ in $X-Z$ plane. The direction of base-plane Poiseuille flow is shown by the arrow along the $X$-direction. (a) Below critical $v_{0}=0.29$ and (b) above $v_{0}=0.29$.

flow is characterised by the appropriate Reynolds number, $R e=U_{c l} h / \nu$, where $U_{c l}$ and $h$ are the experimentally-measured centerline velocity and half-channel height (as previously defined), and $\nu$ the kinematic viscosity.

\section{EXPERIMENTAL RESULTS}

It is known from our previous investigation ${ }^{13}$ using flow visualisation that, when a continuous artificial disturbance (characterised by $v_{0}$ ) is injected into the channel flow, streaks are produced for small values of $v_{0}$, but beyond a critical value of $v_{0}$ (depending upon $R e$ ) hairpin-vortices are shed naturally downstream of the injection slot. Here we present, quantitative measurements of mean velocity profiles and disturbance amplitudes for two different cases. We shall first present results for the 'natural' case where the speaker is not activated, followed by data for phase-locked measurements.

\section{A. Unperturbed or 'natural' streaks and hairpin-vortex formation}

As a preliminary, an example of flow visualisation is shown in figure 3 , where the disturbance (containing smoke particles) is injected into the channel flow, and an image is acquired by a synchronised CCD camera in the $X-Z$ plane for $R e=1660$. The image is captured downstream of the injection slot over the optical window located on the channel floor (see figure 1). Figure 3(a) shows the generation of streaks via a streamwise counterrotating vortex pair (CVP) for small values of $v_{0}$; when $v_{0}$ is increased beyond the critical value of $v_{0}=0.29$ (corresponding to $R e=1660$ ), hairpin-vortices are produced. Figure 3 (b) shows images of hairpin-vortices for $v_{0}$ above the critical value in the $X$ - $Z$ plane.

A hot-wire traversing in $Y$-direction at $Z=0$ and $X=-1.55$ is employed to measure the velocity profiles resulting from the continuous injection of air through the slit at the bottom wall of the channel. Figure 4(a) shows streamwise mean velocity profiles for $v_{0}=0.29$ at various Reynolds numbers. It should be noted that the difference between the theoretical plane Poiseuille flow profile (shown by the solid line) and the measured base-flow profile is a consequence of the flow not being fully developed at this $X$-location. Since for $R e=$ $1660, v_{0}=0.29$ is the critical injection velocity for initiating hairpin-vortices, for any $R e$ below 1660 , the same injection velocity does not produce hairpin-vortices but just a streaky structure (c.f. figure 3a). Accordingly, all the velocity profiles (except at $R e=1660$ ) in figure 4(a) corresponds to streaks.

Inflection in the base-flow profiles indicates a possible source of linear instability with respect to two dimensional waves. Indeed, in these cases, flow visualisations show wavy streaks but without the accompanying hairpin-vortices. Notice that in figure 4(a), with increasing $R e$, the inflection point is associated with increasing shear $(d U / d y)$, which also suggests a stronger unstable flow.

Spectral amplitudes from hot wire measurements at the same $X$ location $(-1.55)$ and at the middle of the shear layer $(Y \approx 0.32)$, which corresponds to the maximum amplitude 

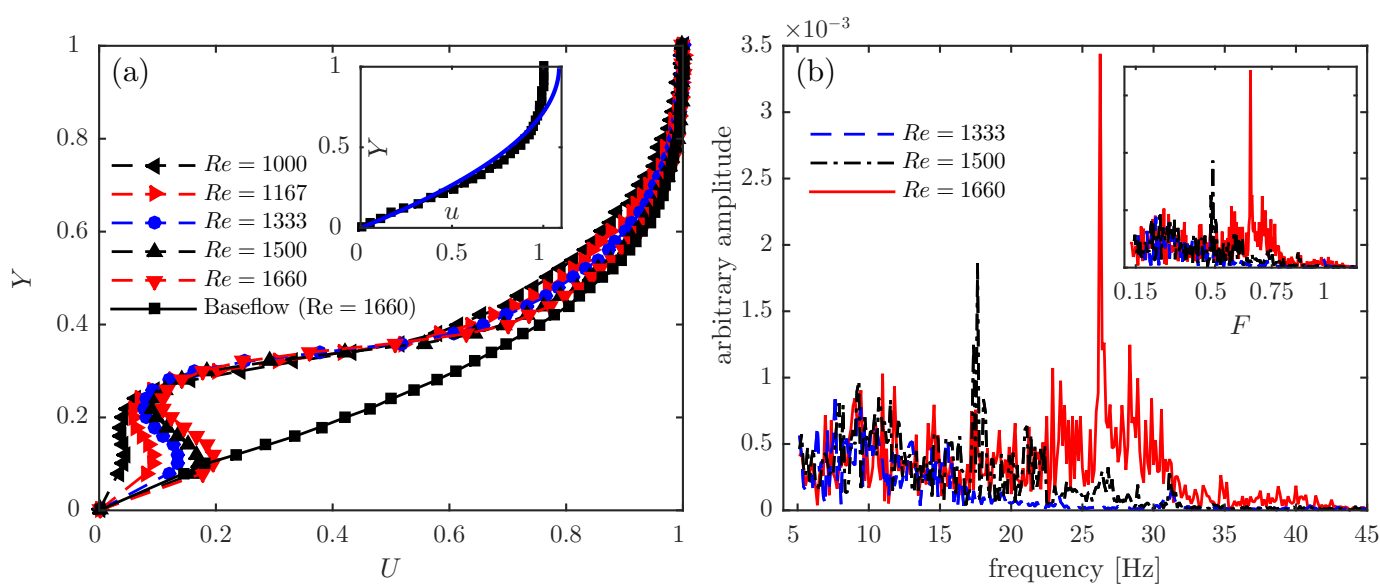

FIG. 4. (Colour online) Hot-wire measurement data at $X=-1.55$ and $v_{0}=0.29$. (a) Time averaged base-flow velocity profiles profiles for various $R e$. The inset shows the experimental baseflow without any jet injection in (black) symbols, which is underdeveloped at the centreline by about $8 \%$ compared to the laminar parabolic velocity profile shown by solid (blue) line at the matched bulk flow rate. (b) Spectral amplitudes corresponding to $R e=1500$ and 1333 at $Y=0.32$; whereas for $R e=1660, Y=0.34$. The inset shows the same figure, however, with the non-dimensional frequency $F=$ frequency $/\left(U_{c l} / h\right)$ on the abscissa.
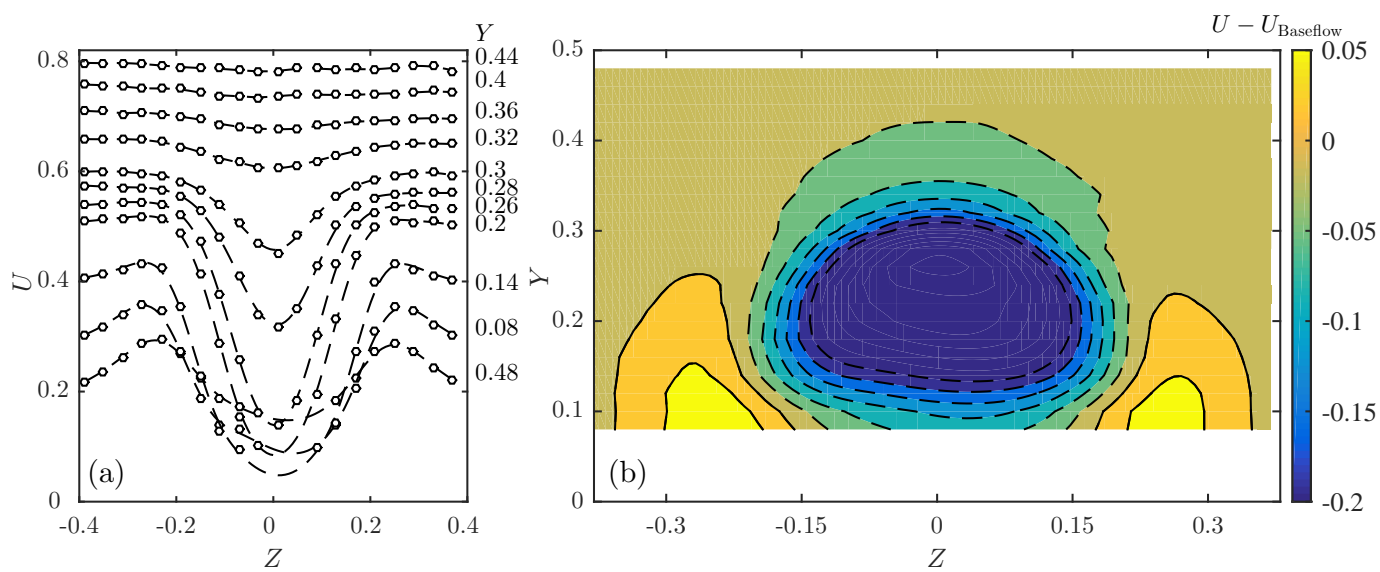

FIG. 5. Base-flow axial velocity profiles from hot-wire anemometry along $Z$-axis for various $Y$ locations at $X=-1.55$, for $R e=1660$ and $v_{0}=0.29$. (a) Total velocity $U$ is plotted along $Z$ and the different $Y$ positions are indicated on the right of the figure. (b) $U-U_{\text {Baseflow plotted on }}$ the $Y-Z$ plane. Here $U_{\text {Baseflow }}$ is taken to be the velocity profile far from the centreline which is approximately same as the velocity profile without any injection, i.e., $v_{0}=0$. Dashed lines show deficit velocity, whereas the solid lines represent excess velocity. The contour values start at 0.05 and decrease in steps of 0.035 till -0.2 .

of the $u$-perturbation for each $R e$ is shown in figure 4(b). It can be observed that the maximum of the relative (arbitrary) amplitude corresponds to a frequency of about $26 \mathrm{~Hz}$ at $R e=1660$ and at reduced frequencies at lower Reynolds numbers. At $R e=1660$, when the hairpin-vortices are shed, the amplitude increases dramatically. At lower $R e$, however, the amplitudes are related to the instability waves associated with the streaks.

As observed in figure 3(a), streaks are nominally streamwise independent, however, they have spanwise variations in their velocity distributions. Typical axial velocity profiles along $Z$ and their distributions in $Y$ are shown in figure 5(a) for $X=-1.55, R e=1660$ and $v_{0}=0.29$. The points show measurements and the dashed lines are spline fit to the points. 
Different $Y$ measurement locations are indicated beside each profile on the right of the figure. To further clarify the flow structure, figure $5(\mathrm{~b})$ shows the velocity profiles from figure 5(a) on $Y-Z$ plane contour plot, after subtracting the mean flow with no injection velocity. The figure shows the central low-speed region flanked by two high speed regions on either sides. The total velocity profiles have a typical wake-like structure with inflection in spanwise direction. Notice that, similar to what was observed in figure 4(a), a careful examination of figure 5 shows that the axial velocity in the wallnormal direction also has an inflectional behaviour.

The strength of the shear at the inflection points play a major role in deciding the spanwise structure of the unstable modes ${ }^{\text {e.g } 28,37}$. Sinuous (anti-symmetric) modes would suggest a dominance of spanwise shear, whereas, varicose (symmetric) modes are assisted predominantly by wallnormal shear. The relative strengths of the spanwise/wallnormal shear depends inversely on the shear layer thickness ${ }^{\text {e.g }} 18$. The thickness of the shear layer for a given profile can be defined as $\delta U /(\partial U / \partial Z)$, where $\delta U$ is the maximum velocity difference along $Z$, and $\partial U / \partial Z$ is the maximum shear in the $Z$-direction.

From figure 5, the spanwise shear layer thickness is $3.4 \mathrm{~mm}$ for $Y=0.08,3.18 \mathrm{~mm}$ for $Y=0.26,4.08 \mathrm{~mm}$ for $Y=0.32$, and likewise. In the wallnormal direction the shear layer thickness is about $1.5 \mathrm{~mm}$ in the $Y$-direction (obtained from figure $4 \mathrm{a}$ ). (We note that, in the wallnormal direction the shear layer has two inflection points as seen in figure 4(a), however, the upper one is more dominating, which we shall observe in the subsequent section in figure 8b). Therefore, the shear layer thickness in $Y$-direction corresponds to this dominating inflection point. Since the absolute growth rate is inversely proportional to the shear layer thickness, it is suggested that the wallnormal inflection will tend to dominate the instability characteristics compared to the spanwise inflection. Furthermore, in the experiments the observation of hairpin-vortices (which are symmetric in spanwise direction) is also indicative of the importance of the wallnormal shear.

\section{B. Phase-locked data}

It was observed in figure 4(b) that at $R e=1660$, the hairpin-vortices are shed at a frequency of about $26 \mathrm{~Hz}$, with a relatively broad spectral noise surrounding this frequency. To reduce the 'jittering' of phases, and to obtain a phase locked data with less noise, it was decided to force the disturbance flow with a very small amplitude of $30 \mathrm{~Hz}$ frequency using a speaker (as described previously in conjunction with figure 2). The selection of the amplitude is made by comparing turbulence levels generated by the speaker and the unforced base-flow, and corresponds to less than $0.2 \%$ of $U_{c l}$. The amplitude where no noticeable difference could be seen between the turbulence levels of the two flows is employed for all the results presented below. It should be mentioned that the sole purpose of the $30 \mathrm{~Hz}$ input disturbance which is close to the shedding frequency of the hairpin-vortices at the same flow conditions is to phase-lock the data and perform more controlled experiments. This in turn after phase-averaging provides a less noisy data which is crucial to obtain smooth eigenfunctions, apart from the other quantities related to the instability wave - to be discussed in the next section.

For obtaining phase-averaged data, we carry out usual triple decomposition ${ }^{38}$ such that the instantaneous velocity $\mathbf{u}$ (PIV map or hot-wire signal) is represented as:

$$
\mathbf{u}=\mathbf{U}+\tilde{\mathbf{u}}+\mathbf{u}^{\prime},
$$

where, $\mathbf{U}$ is the mean, $\tilde{\mathbf{u}}$ is the phase averaged component and $\mathbf{u}^{\prime}$, the remaining fluctuations. Note that, for laminar flow $\mathbf{u}^{\prime}$ is identically zero, and it is close to negligible for our case when we perturb the disturbance flow with the $30 \mathrm{~Hz}$ input frequency. We shall first describe the phase-locked PIV results before presenting hot-wire data. 


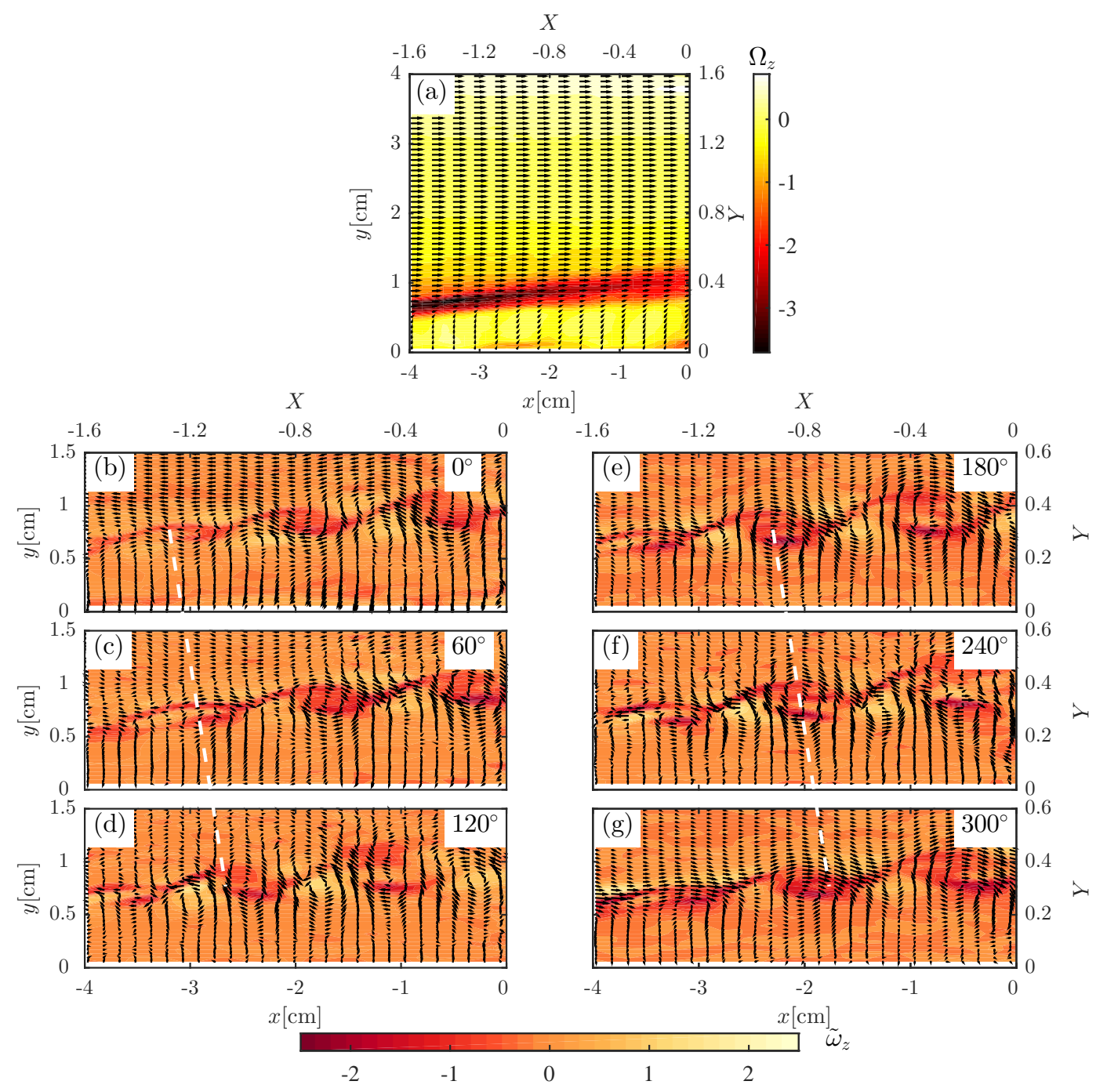

FIG. 6. (Colour online) PIV map at $Z=0$ for $R e=1660$, with $v_{0}=0.29$. (a) Ensemble averaged PIV map. Velocity vectors shown are down-sampled by a factor to two for clarity. (b)-(g) Coherent part of the velocity field (phase averaged PIV map minus the ensemble averaged PIV map) for six different phases separated by $60^{\circ}$.

\section{PIV results}

Figure 6(a) shows velocity vectors and the corresponding vorticity contours defined as, $\Omega_{z}=(\partial V / \partial X-\partial U / \partial Y)$ at $Z=0$ for $R e=1660$ and $v_{0}=0.29$, ensemble averaged over 40 different realisations. The vector map shows that the low momentum fluid is pushed upward by the counter-rotating vortex pair, resulting in the streaky structure shown by the coloured vorticity map of $\Omega_{z}$. In the $X$ direction, the vectors are shown over the injection slot, starting from $X=-1.55$ (i.e $x=-38.75 \mathrm{~mm}$ which is not the leading edge of the injection slot) till $X=0$. Recall that, $X=0$ corresponds to the downstream end of the $57 \mathrm{~mm}$ long injection slot. The vertical range is from $Y=0$ to $Y=1.6$ corresponding to $40 \mathrm{~mm}$ in the wall normal direction. It is noted that $y=50 \mathrm{~mm}$ is the location of the top wall.

The PIV system which is synchronised with the input signal to the speaker measures instantaneous velocity vectors corresponding to the particular phases of the input signal. 


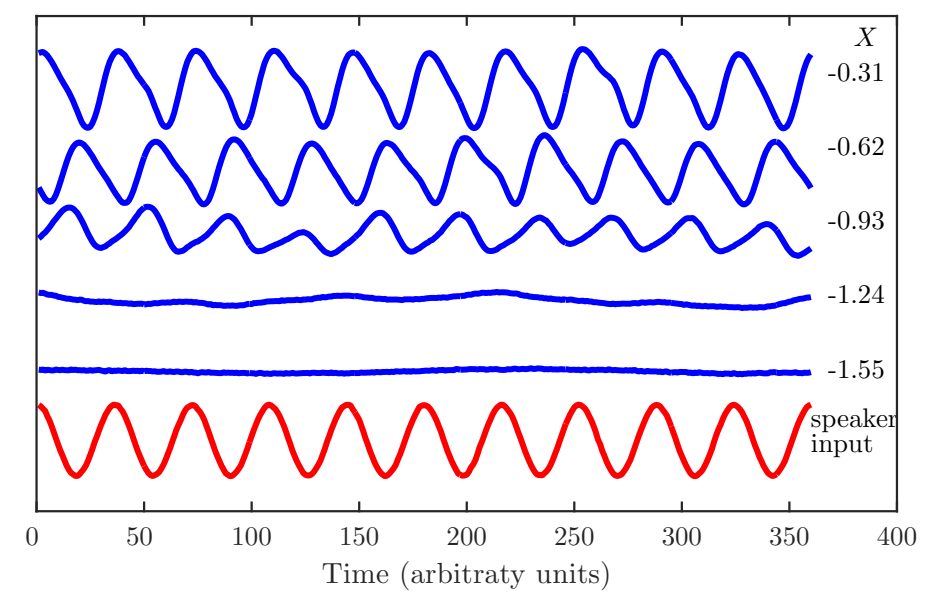

FIG. 7. (Colour online) Input disturbance (at $30 \mathrm{~Hz}$, shown in red, the bottom-most) and phaselocked velocity signals at $R e=1660, Y=0.32$ and $v_{0}=0.29$ for various $X$ locations, increasing from bottom to top. It should be noted that by following a fixed $Y$-location the initial measurement position is slightly above the shear layer.

For each cycle, 36 different velocity measurements are carried out corresponding to a phase difference of $10^{\circ}$ (of the input signal). The initiation of the hairpin-vortices for $R e=1660$ at the critical $v_{0}=0.29$ can be observed quantitatively by PIV when the average base-flow with the streak (figure 6a) is subtracted from a phase averaged vector map. Six such maps of coherent velocity components and their corresponding vorticity with a phase difference of $60^{\circ}$ are shown figure $6(\mathrm{~b})-(\mathrm{g})$. The figure also shows a white dashed line starting from $0^{\circ}$ till $300^{\circ}$ at a slope which corresponds to a distance of $1.6 \mathrm{~cm}$ (the approximate spacing between the hairpin-vortices) in one cycle. The line passes reasonably well through the centre of the vortices for different phases, tracking the development of the vortices.

The cross-section of the hairpin-vortices' heads are clearly visible in the vorticity maps. The velocity vectors also show the crucial role of hairpin-vortices in bringing high momentum from top to bottom and low momentum fluid from bottom to top. This illustrates the high mixing that is evident in turbulent flows (at relatively low Reynolds numbers) which are filled with such hairpin-vortices.

\section{Hot-wire data}

Figure 7 shows the input reference signal to the speaker and phase-locked streamwise velocity signals at various $X$ locations, for $R e=1660, Y=0.32$ and $v_{0}=0.29$. The speaker signal is shown as the bottom-most and the velocity signals are shown from bottom to top with increasing $X$ (marked alongside). With increasing $X$, the velocity signals exhibit oscillations (corresponding to hairpin-vortex shedding) in accordance with the input frequency. Moreover, the magnitudes of the signals grow downstream, reaching an amplitude of about $7 \%$ of $U_{c l}$ at $X=-0.31$. It can also be noticed that the signals undergo change in phase between $X=-0.62$ and -0.31 , indicating approximately half the wavelength or the spacing between hairpin-vortices (which is $\approx(0.62-0.31) 2.5 \mathrm{~cm}=0.775 \mathrm{~cm})$. The lack of oscillations in the first two streamwise positions is due to the fact that at this $X$-location, $Y=0.32$ is slightly above of the shear layer.

The mean velocity profiles measured for $R e=1660$ at various $X$ and $v_{0}=0.29$, perturbed with a small $30 \mathrm{~Hz}$ frequency is shown in figure $8(\mathrm{a})$. With increasing $X$, the thickening of shear layer is noticeable, as was already observed in the PIV map (of figure 6a).

To obtain the coherent part (i.e., eigenfunctions at a specific frequency), the velocity signal at each $X$ is decomposed into a Fourier series and the coefficient at $30 \mathrm{~Hz}$ is extracted. These 

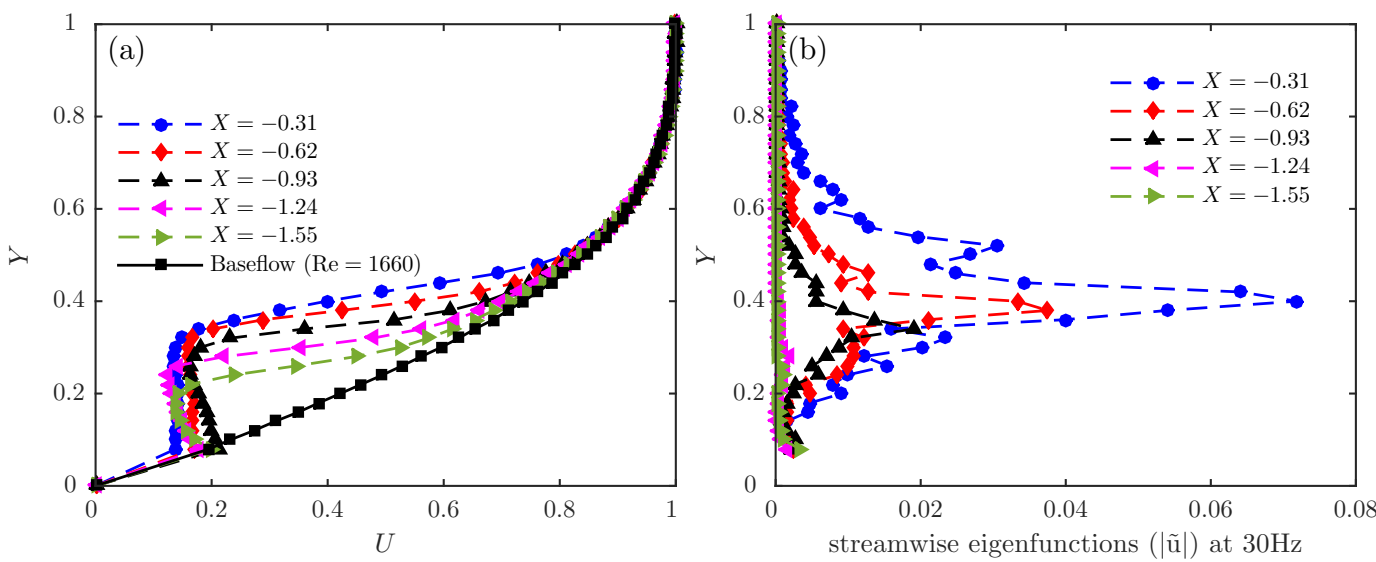

FIG. 8. (Colour online) Hot-wire velocity profile and experimental eigenfunctions at $R e=1660$ and $v_{0}=0.29$, perturbed with a small amplitude wave of $30 \mathrm{~Hz}$. (a) Mean velocity profiles. (b) Experimentally observed streamwise velocity eigenfunctions at various downstream locations calculated at a frequency of $30 \mathrm{~Hz}$.

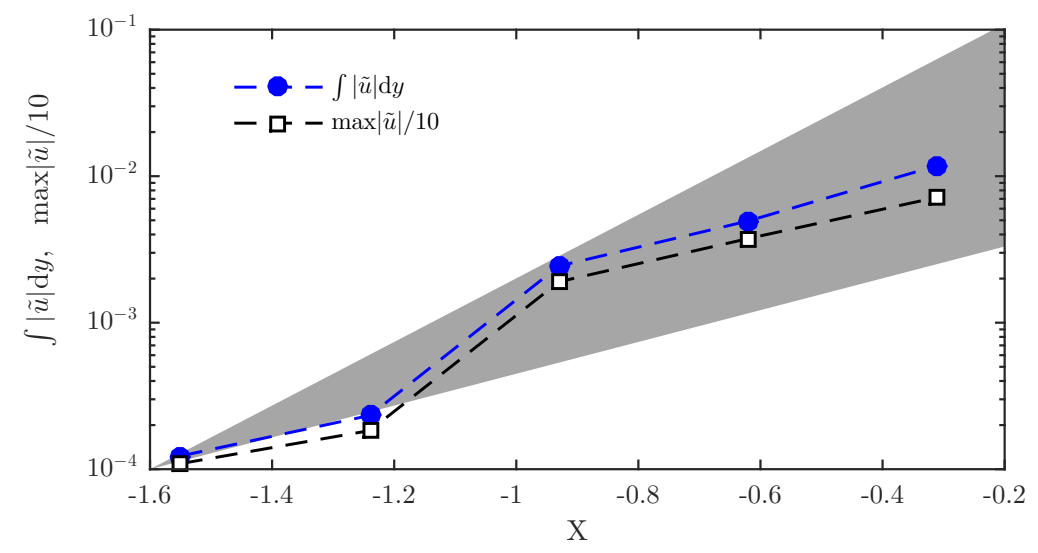

FIG. 9. (Colour online) Experimental growth rate of eigenfunctions (from figure 8b). The shaded grey region is between the lines of slopes (or growth rates) of $2[1 / \mathrm{cm}]$ and $1[1 / \mathrm{cm}]$. Note that, to present the both $\int|\tilde{u}| d Y$ and $\max |\tilde{u}|$ on the same ordinate, the distribution of $\max |u|$ has been divided by a factor of 10 .

coefficients as a function of $Y$, called the eigenfunctions, at various downstream locations are plotted in figure 8(b). They show the typical peak (in $Y$ ) about the inflection point and a rapid growth in the downstream direction. Also observed is the slight shift of the peak away from the wall and widening of the eigenfunctions, both corresponding to the lifting and thickening of the shear layer with $X$ (c.f. figure $6 \mathrm{a}$ ).

The growth in the coherent part of the disturbance or the eigenfunctions can be quantified by monitoring the increase of some characteristic feature of the eigenfunctions, say, $u_{\text {int }}$ with the downstream location $X$. Two common measures for $u_{\text {int }}$ are, (i) the area under the eigenfunction in the $Y$-direction, $\int|\tilde{u}| d Y$, and (ii) the maximum of the eigenfunction (in $Y$ ) at each $X$-location, $\max |\tilde{u}|$. The local exponential growth rate for the experimental data, $\left(\left(-\alpha_{i}\right)_{\text {expt }}\right)$ can be quantified by the expression; $\left(-\alpha_{i}\right)_{\text {expt }}=\mathrm{d}\left(\ln \left(u_{\text {int }}\right)\right) / \mathrm{d} X$. A semilog plot of $\int|\tilde{u}| d Y$ and $\max |\tilde{u}|$ (taken from figure 8b) is presented in figure 9 along the $X$ location. The linear slope of the plot (which is obtained by a least square fit) for $\int|\tilde{u}| d Y$ data is $\left(-\alpha_{i}\right)_{\text {expt }} \approx 1.57[1 / \mathrm{cm}]$, whereas for $\max |\tilde{u}|$, the slope is $\approx 1.47[1 / \mathrm{cm}]$. Notice that, if we consider only the downstream three eigenfunctions, the slope is closer to $1[1 / \mathrm{cm}]$. 

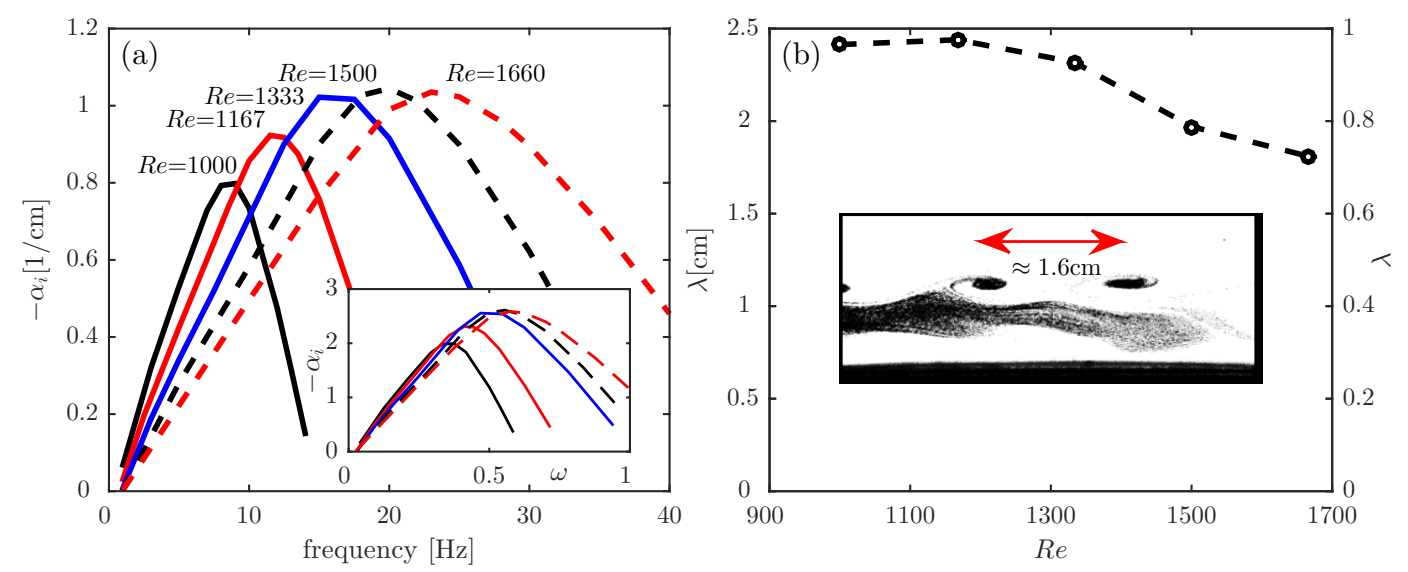

FIG. 10. (Colour online) Stability calculations from the solution of Orr-Sommerfeld equations with $U(Y)$ for $X=-1.55$ at various $R e$ and $v_{0}=0.29$. (a) Growth rate $[1 / \mathrm{cm}] V s$ frequency $[\mathrm{Hz}]$ plot corresponding to the base-flow profiles in figure 4(a). Inset shows the same figure, however, in non-dimensional form. (b) Wavelength $\lambda[\mathrm{cm}]$ Vs $R e$ corresponding to the maximum growth rate in figure $10(\mathrm{a})$. Inset shows the smoke flow visualisation under the same experimental conditions.

\section{LINEAR STABILITY ANALYSIS}

To understand the instability mechanism governing the streak breakdown leading to isolated hairpin-vortices, linear stability analysis of the streaky profiles is performed. The results will be compared with the hot wire anemometry measurements of the velocity signals. For stability analysis, the velocity and pressure fields are decomposed into mean and disturbance fields, such as, $\mathbf{u}(X, Y, Z, t)=\mathbf{U}(Y, Z)+\mathbf{u}_{d}(X, Y, Z, t)$. Here, $\mathbf{U}=(U(Y, Z), 0,0)$ is the known experimental mean flow. The resulting linearised equations (neglecting the nonlinear disturbance terms) governing the disturbance growth are given by:

$$
\begin{aligned}
\frac{\partial u_{d}}{\partial t}+U \frac{\partial u_{d}}{\partial X}+v_{d} \frac{\partial U}{\partial Y}+w_{d} \frac{\partial U}{\partial Z} & =-\frac{\partial p_{d}}{\partial X}+\frac{1}{R e} \nabla^{2} u_{d} \\
\frac{\partial v_{d}}{\partial t}+U \frac{\partial v_{d}}{\partial X} & =-\frac{\partial p_{d}}{\partial Y}+\frac{1}{R e} \nabla^{2} v_{d} \\
\frac{\partial w_{d}}{\partial t}+U \frac{\partial w_{d}}{\partial X} & =-\frac{\partial p_{d}}{\partial Z}+\frac{1}{R e} \nabla^{2} w_{d} \\
\frac{\partial u_{d}}{\partial X}+\frac{\partial v_{d}}{\partial Y}+\frac{\partial w_{d}}{\partial Z} & =0
\end{aligned}
$$

where, $p_{d}$ is the disturbance pressure incorporating the density factor.

Even though the mean velocity $U$ is a function of both $Y$ and $Z$, we know that the magnitude of $\partial U / \partial Y$ is larger than $\partial U / \partial Z$ (c.f. discussion related to figure 5 ). Therefore, at the first instance, we shall neglect the term $\partial U / \partial Z$ from (2a) in sections IV A and IV B, and in IV C include the spanwise shear. Furthermore, the velocity measurements are made without an external forcing of the disturbance (by the speaker) and with the forcing. First, the results obtained with no external forcing are discussed.

\section{A. Linear stability based on $U(Y)$ for the unperturbed or 'natural' case}

With $U(Y)$, the disturbance field can be represented as, $\mathcal{R} e\{\boldsymbol{q}\}=\left(u_{d} ; v_{d} ; w_{d} ; p_{d}\right)$, where, $\mathcal{R} e\{\ldots\}$ is the real part, and,

$$
\boldsymbol{q}=\hat{\boldsymbol{q}}(y) \mathrm{e}^{\mathrm{i}(\alpha x-\omega t)}
$$


For the spatial stability analysis which we perform, $\omega$ is real and $\alpha=\alpha_{r}+\mathrm{i} \alpha_{i}$ is a complex number. Substituting (3) into (2) results in (after excluding $\partial U / \partial Z$ ) the Orr-Sommerfeld equation (for 2D, parallel base-flow). The resulting partial differential equations are converted to algebraic ones and discretised by employing 120 Chebychev polynomials. The final generalised eigenvalue problem is solved using the commercial software MATLAB.

For the 'unperturbed' case considered in this section, the Orr-Sommerfeld equation is solved for the base-flow profiles $U(Y)$, which are shown in figure 4(a). The velocity profiles are measured from $Y=0.08$ till $Y=1.08$ (with $Y=0.08$ to $Y=0$ assumed linear). The velocity of the rest of the $Y$-locations are obtained by reflecting a profile from the bottom half of the channel when no disturbance is injected. A sample measurement with the streaks has shown that there is no noticeable effect of streaks on the velocity profile in the top half for the $X$-locations considered.

Figure 10(a) shows the maximum growth rate $\left(-\alpha_{i}\right.$, in $\left.[1 / \mathrm{cm}]\right)$ for various input frequencies (given in $[\mathrm{Hz}]$ ) at different $R e$. The growth rate increases with increasing $R e$ and for $R e$ above 1333 the maximum growth saturates. Nevertheless, hairpin-vortices are first observed at $R e=1660$ with a frequency corresponding to the maximum growth rate about $24 \mathrm{~Hz}$. Furthermore, the frequencies corresponding to the peaks in growth rates for $R e=1660$ and 1500 in figure 10(a) are in the range of the experimentally observed spectral peaks in figure 4(b) for the corresponding Reynolds numbers.

For each $R e$ in figure 10(a), the $\alpha_{r}$ corresponding to the peak in $-\alpha_{i}$ is plotted in figure 10 (b) as a wavelength, $\lambda=2 \pi / \alpha_{r}$, which is 'the most amplified wave' according to the linear theory. Figure 10(b) also shows experimentally observed flow visualisation and the corresponding wavelength. In the theoretical predictions, the value of $\lambda$ decreases with increasing $R e$, and for $R e=1660$ the predicted $\lambda=1.8 \mathrm{~cm}$, whereas the experimentally observed one is about $1.6 \mathrm{~cm}$.

The agreement between the linear theory and experiments is reasonably good, however, it is not possible to understand the region of the flow where the instability is dominant without a phase-locked data, which we carry out in the following.

\section{B. Linear stability based on $U(Y)$ for the perturbed or 'phase-locked' case}

Stability calculations follow the same procedure as in the previous section, except that here we employ $U(Y)$, which are obtained from the hot-wire phase-locked measurements presented in figure $8(\mathrm{a})$. Recall that in figure $8(\mathrm{a})$ all the velocity profiles correspond to a single $R e=1660$, however, for increasing $X$.

As in the previous section, the wavelengths of the most amplified waves according to the linear stability theory are shown in figure 11a. It shows that the predicted wavelength increases with $X$ and reaches a value of $1.62 \mathrm{~cm}$ at $X=-0.31$. This is not surprising because with increasing $X$ the shear layer thickens slightly and the most amplified wavelengths are proportional to the shear-layer thickness. The experimentally observed value of the wavelength (as shown in the inset of the figure 11a) is about $1.6 \mathrm{~cm}$, comparing well to that obtained from stability calculations.

The main advantage of using the phase-locked data is in the ability to make a comparison of the eigenfunctions calculated from the stability analysis and that observed in the experiments. Figure 11(b) shows the normalised streamwise eigenfunctions both theoretically and experimentally for a specific $X$. Theoretically obtained eigenfunction at $X=-0.62$ is shown and is compared with that found experimentally at the same location (from figure $8 \mathrm{~b}$ ), with a very good agreement between the two. The maxima of the eigenfunctions match well with the locations of inflections in the base-flow profile, which shows that the instability is of inviscid type based on the wallnormal inflectional profile.

Furthermore, the growth rates obtained from the linear stability are about $1[1 / \mathrm{cm}]$. When compared with the experimental growth rates presented in figure 9 , the linear stability predictions are slightly less (or even comparable, if we consider the downstream three growing experimental eigenfunctions). We recall that the conditions of our experiments are such that 

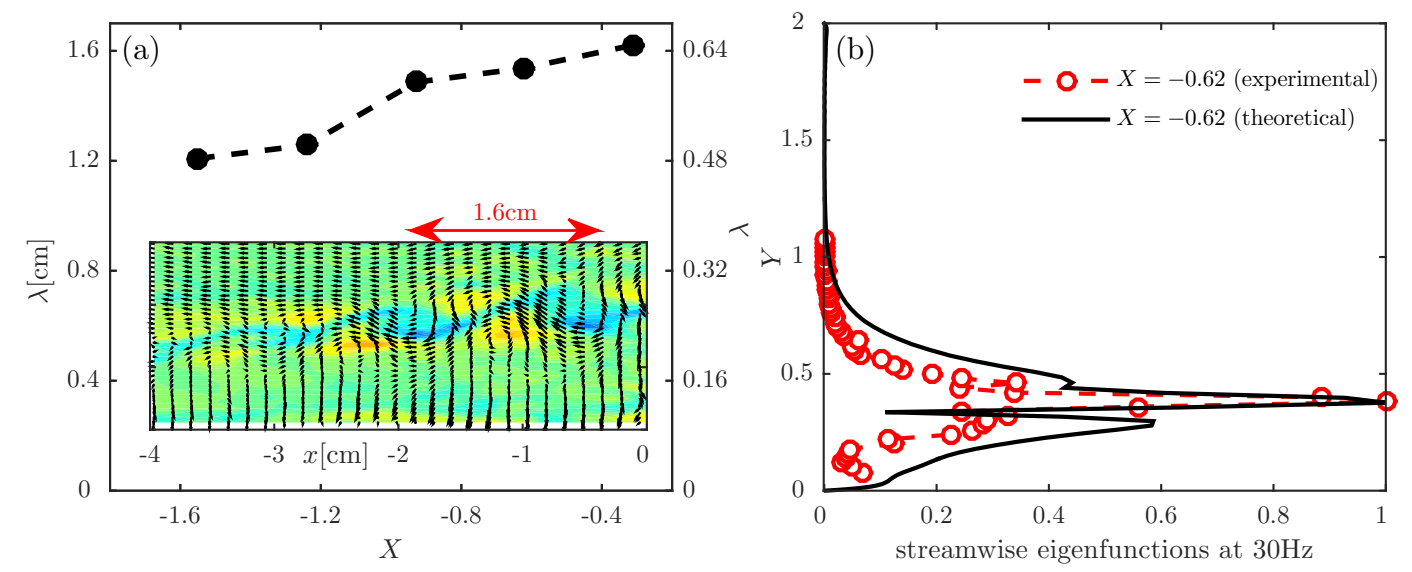

FIG. 11. (Colour online) Stability calculations from the solution of Orr-Sommerfeld equations with $U(y)$ for $R e=1660$ at various $X$ and $v_{0}=0.29$, with velocity profiles from the phase-locked experiments. (a) Theoretical predictions and comparison to experimental results of the wavelength for various $X$ locations at $30 \mathrm{~Hz}$. The $\lambda V s X$ plot has an inset which shows the PIV map (from figure 6) comparing the experimentally observed wavelength. (b) Comparison of experimentally observed eigenfunction of streamwise velocity and from stability calculations.

the streak has become unstable and hairpin-vortices are being generated. In this connection, it interesting to note that during transition to turbulence in a boundary layer accompanied by the generation of hairpin-vortices, the growth of $u$-fluctuations exhibits large growth rates (shown by figure 22 of Klebanoff et al..$^{4}$ ), which is higher than that which can be predicted by instability mechanism using the corresponding inflectional base-flow ${ }^{39}$.

\section{Linear stability based on $U(Y, Z)$ for the unperturbed or 'natural' case}

The mean flow $U(Y, Z)$ is essentially two dimensional despite the fact that we have used $U(Y)$ in the above calculations. Even though the analysis with $U(Y)$ is based on reasonable observations that the wallnormal shear was noted to be dominant over the spanwise shear, a $U(Y)$-based analysis is not capable of pointing out the exact role of $\partial U / \partial Z$ vis-a-vis $\partial U / \partial Y$. Furthermore, $U(Y)$ is incapable of showing the spanwise structure of the eigenmodes to determine if the most unstable modes are varicose or sinuous types. Consequently, we carry out a stability analysis with an experimentally measured $U(Y, Z)$ - shown in figure 5 . We recall that figure 5 was obtained without phase-lock, and here serves us primarily to determine the relative importance of $\partial U / \partial Y$ and $\partial U / \partial Z$ on the stability characteristics.

\section{Formulation of the eigenvalue problem}

To perform the analysis the experimental velocity field has been decomposed into Fourier series in the spanwise direction:

$$
U(Y, Z)=\sum_{n=0}^{N} F_{n}(Y) \cos (n \beta Z),
$$

where $\beta$ is the spanwise wavenumber deduced from figure $5(\beta=2 \pi / 0.783=8.024)$. Several values of the cutoff harmonic $N$ were compared and it has been found that $N=2$ (which results in three terms in the Fourier expansion) is sufficient to obtain the eigenvalues within accuracy of $1 \%$. According to Floquet theory with respect to the spanwise direction a 

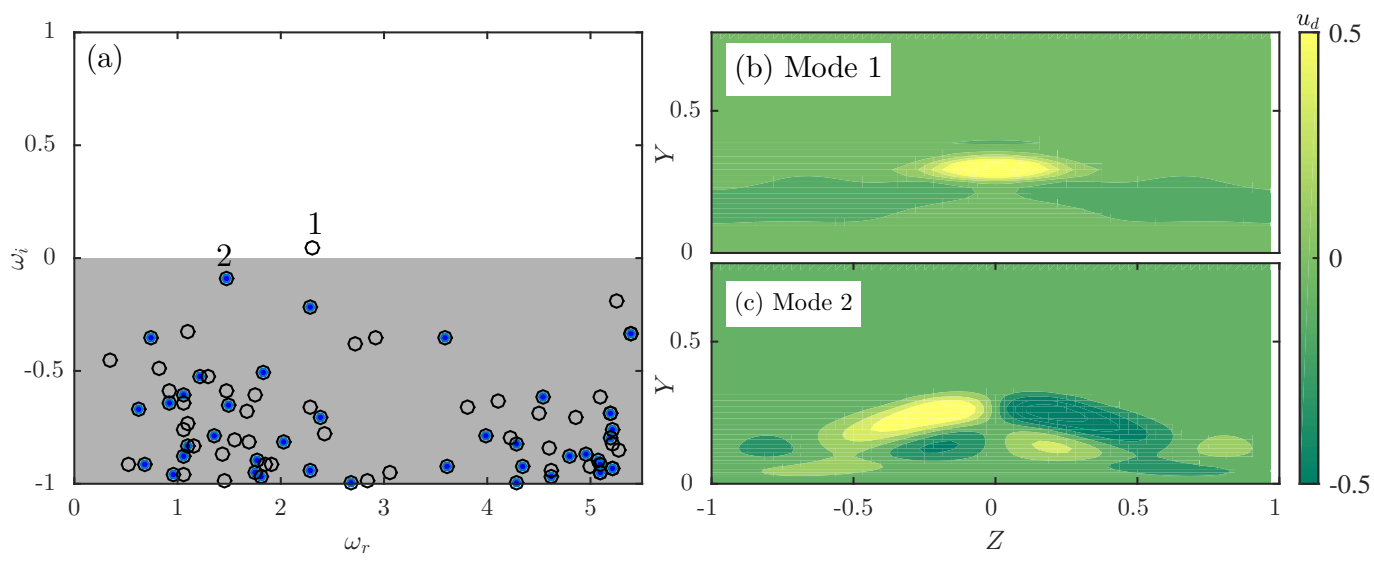

FIG. 12. (Colour online) Temporal stability results for $\alpha=2.5[1 / \mathrm{cm}]$ (the heavy $\boldsymbol{\Delta}$ symbol in figure 14). (a) Growth rate versus temporal frequency plot. The empty symbols are for the varicose modes whereas the filled ones are for the sinuous modes. The two least stable modes are marked with numbers 1 and 2. (b) and (c): Streamwise eigenfunction $\left(u_{d}\right)$ for the eigenmodes marked 1 and 2 , respectively in (a). The symmetric features (about $Z=0$ ) for mode 1 shows its varicose characteristics, where the anti-symmetric shape of mode 2 is indicative of its sinuous nature.

secondary disturbance of the following form is considered:

$$
\boldsymbol{q}=e^{i(\alpha x-\omega t)} \sum_{k=-M_{z}}^{M_{z}} \hat{\boldsymbol{q}}_{k}(Y) e^{i \beta k Z},
$$

where, $\mathcal{R} e\{\boldsymbol{q}\}=\left(u_{d} ; v_{d} ; w_{d} ; p_{d}\right), \omega$ is its real (complex) frequency and $\alpha$ is the complex (real) wavenumber for spatial (temporal) stability analysis. It should be noted that $\beta$ is associated with the modified base-flow, whereas $\omega, \alpha$ are associated with the secondary disturbance. Although the summation in (5) should include infinite number of terms, for numerical purposes, the summation is performed up to $k=M_{z}$. Furthermore, in (5) we consider the usual case of fundamental mode, which implies that we set the detuning parameter equal to zero ${ }^{18}$.

In the literature, it is usual to carry out temporal stability analysis ${ }^{18}$, which in most cases is sufficient to extract the physics of the problem. We, however, have a spatially evolving flow and a spatial stability analysis would be more appropriate. Therefore, we first carry out a temporal stability, which will convey the main message, and subsequently, we show the spatial analysis which confirms our conclusions from temporal calculations.

For stability analysis (5) along with (4) are substituted into (2) resulting in an eigenvalue problem. For temporal stability, $\omega=\omega_{r}+\mathrm{i} \omega_{i}$ (with $\omega_{r}$ and $\omega_{i}$ the temporal frequency and growth rate, respectively) and $\alpha$ is a real streamwise wavenumber, and the resulting equations are provided in Appendix C of Karp and Cohen ${ }^{29}$. On the other hand, for spatial stability, $\omega$ is a real number and $\alpha=\alpha_{r}+\mathrm{i} \alpha_{i}$ (with $\alpha_{r}$ and $-\alpha_{i}$ the spatial wavenumber and growth rate, respectively), and the resulting equations are given in Appendix A of this paper.

\section{Results for the temporal and spatial stability analysis of $U(Y, Z)$}

In the following, we focus primarily on the temporal stability analysis which shows less ambiguously the effects of wallnormal and spanwise shears on the least stable mode. Further details in terms of the unstable modes from both temporal and spatial stability of $U(Y, Z)$ are presented in Appendix B. 

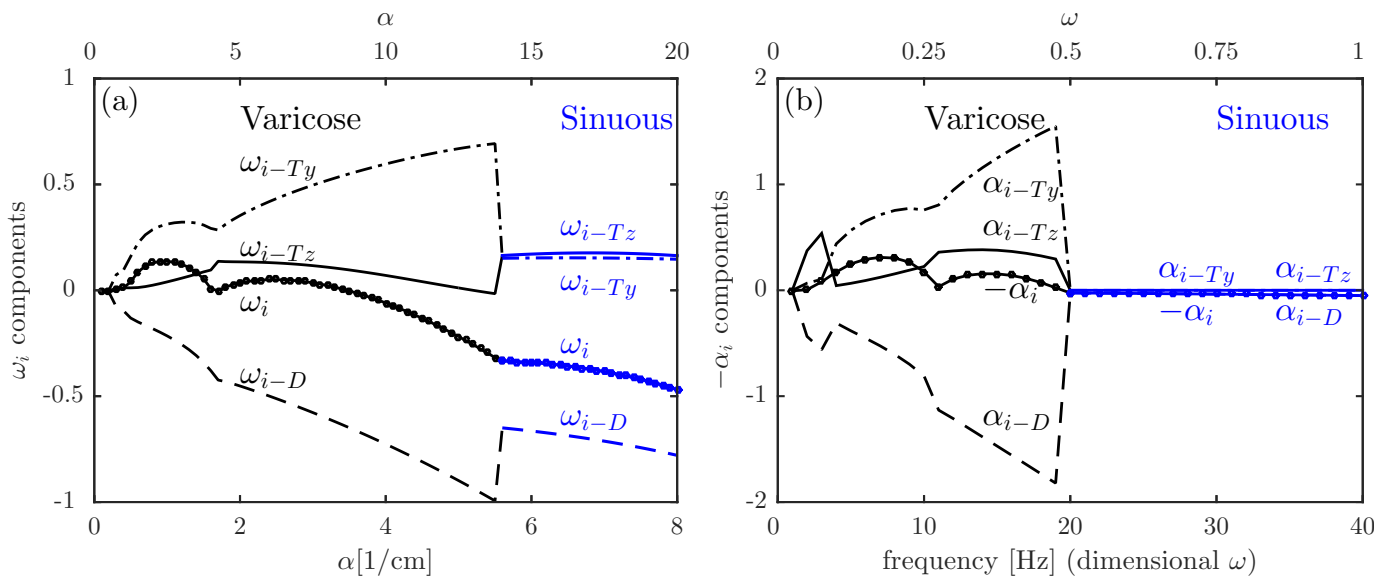

FIG. 13. (Colour online) Relating growth rates to energy balance. (a) Energy balance for temporal stability analysis. (b) Energy balance for spatial stability analysis.

For the temporal case (as well as in spatial case), all unstable modes of $U(Y, Z)$ are of symmetric, varicose type. To highlight the common features of these modes, we select $\alpha=2.5[1 / \mathrm{cm}]$ (the heavy $\boldsymbol{\Lambda}$ symbol in figure 14 ), which corresponds to a particular unstable mode. Figure 12(a) shows all the modes for $\alpha=2.5[1 / \mathrm{cm}]$ on a complex $\omega$ plane. The unstable mode is marked 1 , and the corresponding eigenfunction is presented in figure 12(b), which shows unambiguously that the mode is a symmetric, varicose one. Interestingly, the second least stable mode is a sinuous one marked 2, with the corresponding eigenfunction presented in $12(\mathrm{c})$. Note that since the velocity measurements in figure 5 are not phaselocked, unfortunately no comparison to experimental eigenfunctions is possible like in the case of stability analysis with $U(Y)$.

The physical reason for the instability can be obtained by investigating the different contributions to the 'disturbance' energy equation. For the temporal case $e^{\text {e.g., }}, 37,40$ the different physical mechanisms contributing to the growth rates can be identified:

$$
\omega_{i}=\omega_{i-T y}+\omega_{i-T z}+\omega_{i-D}
$$

where, $\omega_{i-T y}$ and $\omega_{i-T z}$ are related to the shear productions due to $d U / d Y$ and $d U / d Z$, respectively, whereas $\omega_{i-D}$ is the contribution from viscous dissipation. Similar explicit expression for these terms have been previously derived for temporal case ${ }^{\text {e.g., } 37,40}$, and we present them in Appendix C 1 for our case of plane Poiseuille flow.

The results for the temporal case is presented in figure 13(a). Note that the distribution of $\omega_{i}$ is same as that shown in the Appendix B, figure 14(b). For each least stable mode the three different components on the right-hand-side of (6) are calculated. For lower wavenumbers symmetric varicose modes are least stable and for higher wavenumbers the anti-symmetric sinuous modes dominate, however, only the varicose modes are unstable. This is consistent with the experiments. For the varicose mode, $\omega_{i-T z}$ is relatively small and the main balance is between $\omega_{i-T y}$ and $\omega_{i-D}$, corroborating the results from previous investigations ${ }^{28,37,40}$. For the case of sinuous modes $\omega_{i-T z}$ becomes larger than $\omega_{i-T y}$ and both in turn balance the viscous forces. The viscous effects, however, dominates the shear in the sinuous case and we have only damped modes.

For a spatially evolving flow, like in our case, it is more appropriate to consider spatial analysis. The results have close similarities to the temporal case (as presented in Appendix B). Furthermore, to clarify the physical mechanism, similar to energy analysis in the temporal case (c.f. (6)), we can derive an expression for $\alpha_{i}$ :

$$
-\alpha_{i}=\alpha_{i-T y}+\alpha_{i-T z}+\alpha_{i-D},
$$

the details of which are provided in Appendix C 2. Here, again, $\alpha_{i-T y}$ and $\alpha_{i-T z}$ are related to $d U / d Y$ and $d U / d Z$ shears respectively, and $\alpha_{i-D}$ to dissipation. Figure 13(b) shows the 
results, and again, only varicose modes are unstable which are dominated by the wallnormal shear production. The situation of sinuous modes is less transparent in the spatial case, however, it is clear that wallnormal shear does not dominate.

Both the temporal and spatial energy analyses suggest the dominance of $d U / d Y$, which was indeed observed during the calculations of shear layer thickness with regard to figure 5 . This was further strengthened by the 1D stability analysis in which the spanwise variations of the base-flow were ignored, and good agreement with the measurements was obtained. Unfortunately, a detailed comparison of $2 \mathrm{D}$ stability analysis with the experiments (as in $1 \mathrm{D}$ stability) is not possible because the $2 \mathrm{D}$ velocity measurements are not phase-locked.

\section{SUMMARY AND CONCLUSIONS}

Jet-in-crossflow is a fundamental flow scenario with ample technological applications. The flow structures include streak and hairpin-vortices, and there is much interest in understanding the generation mechanism of these coherent structures. Furthermore, streaks and hairpin-vortices are the key organised motions of transitional/turbulent flows and there has been increasing interest in artificially creating them, both for the purpose of understanding the basic mechanism ${ }^{28}$, as well as to control the flow ${ }^{41}$. However, it is well known that the streaks usually are most unstable to sinuous modes ${ }^{18,42}$, and therefore creating streaks that will naturally become unstable to varicose modes rather than the sinuous modes are not easy. Also, very few investigations have focused on experiments, which are important to carry forward our understandings to applications. As such, the present set of experiments provide a robust way of artificially producing streaks though a streamwise elongated slotted jet in channel crossflow, and subsequently studying their natural instability to symmetric varicose modes and further hairpin-vortex formation, utilising tools from linear instability and concomitant experimental techniques.

Experiments are conducted in a plane-Poiseuille crossflow facility with air as the working medium. The jet is issued into the channel flow through an almost independent streamwise slot, which for a range of injection velocities $v_{0}$ produce streaks and, with increasing $v_{0}$ become unstable and breakdown to hairpin-vortices. The flow field is visualised using smoke and measured using the techniques of PIV and hot-wire anemometry. Both 'natural' and phase-locked data is generated. There is a clear evidence of streak instability with increasing $R e$ and further breakdown. The phase-locked PIV data is able to show the evolution of the coherent part of the disturbance with downstream distance. The hot-wire data has been utilised to extract the eigenfunctions and growth-rates of the unstable wave.

Subsequently, Orr-Sommerfeld equation is solved for the case where base-flow is 1D, $U(Y)$, both for the natural as well as for the case of phase-locked scenario. The most unstable mode has a wavelength which is close to that observed experimentally, and the good agreement between the experimental and theoretical eigenfunctions are obtained. These suggest the dominance of wallnormal shear in our experimental setup which eventually leads to the generation of hairpin-vortices. The growth rates predicted by the linear stability is slightly lower than that observed experimentally, similar to the case of a 'real' transition experiment ${ }^{4,39}$. In that regards, our artificial streaks behave very much the same way as those observed in transitional flows. Furthermore, the stability analysis of the experimentally obtained $2 \mathrm{D}$ base-flow $U(Y, Z)$ is carried out using the usual Floquet method for both the temporal and spatial cases, along with an energy balance analysis to isolate the physical mechanism responsible for the instability. They point out the dominance of wallnormal shear $d U / d Y$ over the spanwise shear $d U / d Z$ in producing the symmetric varicose modes, which are precursors to the fully developed symmetric structure found in the hairpin-vortices. All the anti-symmetric modes are found to be damped, in agreement with the experiments, which when found to be least stable were characterised by a larger $d U / d Z$. 


\section{ACKNOWLEDGMENTS}

This research has been supported by the Israeli Science Foundation under Grant no. 1247/06. The authors are grateful to Efim Shulman and Oleg Kan for their help in constructing the apparatus and LabView ${ }^{\mathrm{TM}}$ programming. JP gratefully acknowledges support from the Australian Research Council.

\section{Appendix A: Secondary stability analysis}

This appendix describes the derivation of the secondary stability analysis. Substituting the disturbance (5) into the linearised Navier-Stokes equations (2), with the aid of the following relations $(m=1,2)$ :

$$
\begin{aligned}
\sum_{k} \hat{\boldsymbol{q}}_{k}(Y) \cos (m \beta Z) e^{i \beta k Z} & =\sum_{k} \frac{\hat{\boldsymbol{q}}_{k-m}(Y)+\hat{\boldsymbol{q}}_{k+m}(Y)}{2} e^{i \beta k Z}, \\
\sum_{k} \hat{\boldsymbol{q}}_{k}(Y) \sin (m \beta Z) e^{i \beta k Z} & =\sum_{k} \frac{\hat{\boldsymbol{q}}_{k-m}(Y)-\hat{\boldsymbol{q}}_{k+m}(Y)}{2 i} e^{i \beta k Z},
\end{aligned}
$$

results in the following eigenvalue problem for the calculation of $\alpha$ :

$$
\left(\begin{array}{cccccc}
O & -D_{y} & -D_{z} & O & O & O \\
O & O & O & O & I & O \\
O & O & O & O & O & I \\
A & V D_{y}-V_{y} & V D_{z}-V_{z} & O & -\frac{1}{R e} D_{y} & -\frac{1}{R e} D_{z} \\
O & -\operatorname{ReA} & O & R e D_{y} & \operatorname{ReV} & 0 \\
O & O & -\operatorname{ReA} & \operatorname{Re} D_{z} & O & \operatorname{ReV}
\end{array}\right)\left\{\begin{array}{c}
\tilde{u} \\
\tilde{v} \\
\tilde{w} \\
\tilde{p} \\
\tilde{v}_{x} \\
\tilde{w}_{x}
\end{array}\right\}=i \alpha\left\{\begin{array}{c}
\tilde{u} \\
\tilde{v} \\
\tilde{w} \\
\tilde{p} \\
\tilde{v}_{x} \\
\tilde{w}_{x}
\end{array}\right\}
$$

where $I$ is the identity matrix, $O$ is a zero matrix, $\tilde{u}$ (and similarly $\tilde{v}, \tilde{w}, \tilde{p}$ ) is defined by:

$$
\tilde{u}=\left(\hat{u}_{-M_{z}}, \hat{u}_{-\left(M_{z}-1\right)}, . ., \hat{u}_{M_{z}}\right),
$$

and the matrices $A, V, V_{y}, V_{z}, D_{y}$ and $D_{z}$ are defined by:

$$
\begin{gathered}
A=\left(\begin{array}{ccccc}
a_{-M_{z}} & O & . & O \\
O & a_{-\left(M_{z}-1\right)} & O & . . \\
. \ddot{O} & & . . & \ddot{O} & . . \\
a_{M_{z}}
\end{array}\right), \\
V=\frac{1}{2}\left(\begin{array}{cccccc}
2 F_{0} & F_{1} & F_{2} & O & . . & O \\
F_{1} & 2 F_{0} & F_{1} & F_{2} & O & . . \\
F_{2} & F_{1} & 2 F_{0} & F_{1} & F_{2} & O \\
O & F_{2} & F_{1} & 2 F_{0} & F_{1} & . . \\
. . & . . & . . & . . & . . & . . \\
O & . . & O & F_{2} & F_{1} & 2 F_{0}
\end{array}\right), \\
V_{y}=\frac{1}{2}\left(\begin{array}{cccccc}
2 F_{0}^{\prime} & F_{1}^{\prime} & F_{2}^{\prime} & O & . . & O \\
F_{1}^{\prime} & 2 F_{0}^{\prime} & F_{1}^{\prime} & F_{2}^{\prime} & O & . . \\
F_{2}^{\prime} & F_{1}^{\prime} & 2 F_{0}^{\prime} & F_{1}^{\prime} & F_{2}^{\prime} & O \\
O & F_{2}^{\prime} & F_{1}^{\prime} & 2 F_{0}^{\prime} & F_{1}^{\prime} & . . \\
. & . . & . . & . . & . . & . . \\
O & . . & O & F_{2}^{\prime} & F_{1}^{\prime} & 2 F_{0}^{\prime}
\end{array}\right),
\end{gathered}
$$




$$
\begin{gathered}
V_{z}=\frac{\beta}{2 i}\left(\begin{array}{cccccc}
O & F_{1} & 2 F_{2} & O & . . & O \\
-F_{1} & O & F_{1} & 2 F_{2} & O & . . \\
-2 F_{2} & -F_{1} & O & F_{1} & 2 F_{2} & O \\
O & -2 F_{2} & -F_{1} & O & F_{1} & . . \\
. . & . . & . . & . . & . . & . . \\
O & . . & O & -2 F_{2} & -F_{1} & O
\end{array}\right), \\
D_{y}=\left(\begin{array}{cccc}
D & O & . . & O \\
O & D & O & . . \\
. . & . . & . . & . . \\
O & . . & O & D
\end{array}\right),
\end{gathered}
$$

$$
D_{z}=i \beta\left(\begin{array}{cccc}
-M_{z} I & O & . . & O \\
O & -\left(M_{z}-1\right) I & O & . . \\
. . & . . & . . & . . \\
O & . . & O & M_{z} I
\end{array}\right)
$$

where $D$ is the matrix that represents derivation with respect to the $Y$ coordinate and $a_{n}$ is defined by:

$$
a_{n}=\left(i \omega-\frac{(n \beta)^{2}}{R e}\right) I+\frac{1}{R e} D^{2} ; \quad n=-M_{z}, . ., M_{z} .
$$

The eigenvalue problem is solved numerically using MATLAB software with 101 Chebyshev modes in the wallnormal direction. The simple harmonic dependence of the base-flow upon the spanwise coordinate allows convergence for relatively small values of $M_{z}$ and it is found that $M_{z}=5$ is sufficient in order to obtain the eigenvalues within an accuracy of $0.5 \%$.

\section{Appendix B: Further results from the stability analysis of $U(Y, Z)$}

The temporal stability analysis of $U(Y, Z)$ from figure 5 are presented in figure 14 . The frequencies $\left(\omega_{r}\right)$ and the corresponding growth rates $\left(\omega_{i}\right)$ for the least stable modes are shown in dimensional forms in Figures 14(a) and (b), respectively. It is evident from the growth rate distributions that the unstable modes are of varicose type (empty symbols), and they correspond to frequencies which are comparable to the ones observed experimentally. Only for higher wavenumbers does the sinuous modes (full symbols) became least stable, and all of them are damped, with much lower frequencies. We note that, when compared to temporal stability results of the 1D base-flow $U(Y)$ (not shown here), the frequencies are comparable, whereas the growth rates in the 1D cases are higher.

Figures 15(a) and (b) present least stable modes from the spatial stability analysis of $U(Y, Z)$ from figure 5. The results have similarities to the temporal case, in that the unstable modes are again of the varicose type, whereas all the sinuous type modes are damped. The wavenumber $\left(\alpha_{r}=2 \pi / \lambda\right) 3.5[1 / \mathrm{cm}]$ corresponds to a varicose mode with wavelength of about $1.8 \mathrm{~cm}$, which is close to the experimentally observed ones. This mode also shows a frequency which is approximately similar to the experimental ones.

\section{Appendix C: Evolution of perturbation kinetic energy for $U(Y, Z)$}

\section{Temporal stability}

For temporal stability analysis the equation governing the growth rate is similar to previous studies ${ }^{\text {e.g. }}, 37,40$, except that in our case of plane-Poiseuille flow the wallnormal integration is from -1 to 1 . Succinctly, the procedure is to derive the disturbance kinetic energy 

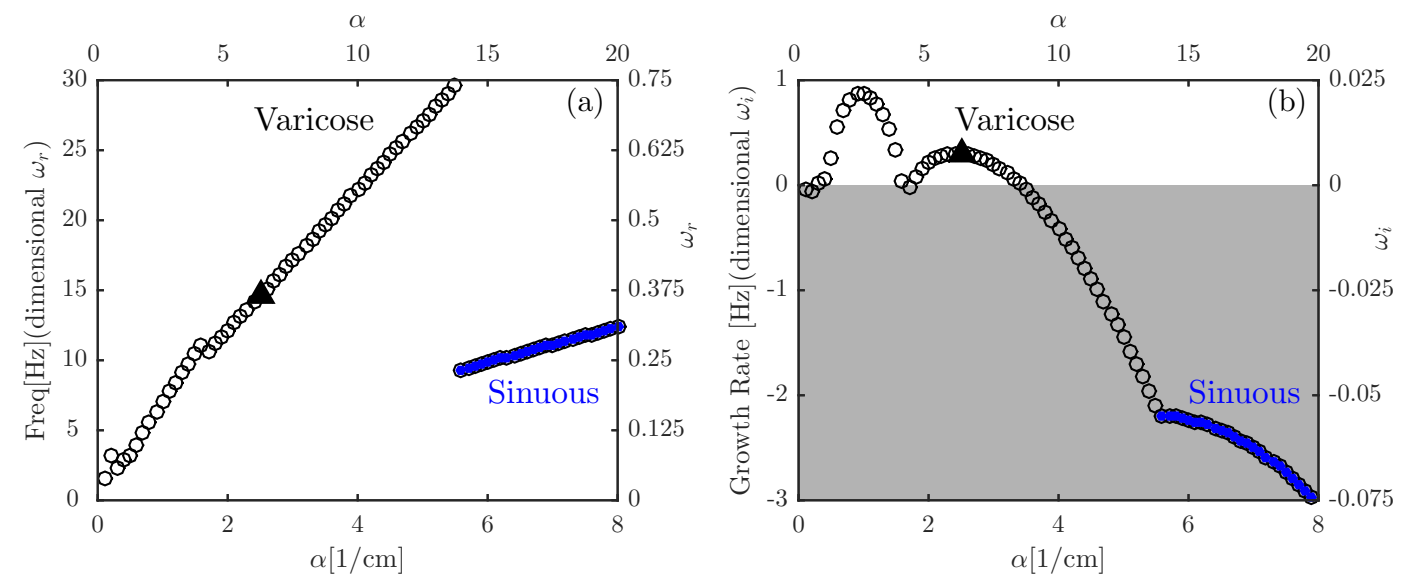

FIG. 14. (Colour online) Temporal stability analysis: least stable eigenvalues for the base-flow $U(y, z)$ in figure 5. The empty circular symbols are for the varicose modes whereas the filled circular symbols are for the sinuous modes. (a) The temporal frequency $\left(\omega_{r}\right)$ in dimensional form corresponding to the least stable modes. The heavy $\boldsymbol{\Delta}$ symbol denotes $\alpha=2.5[1 / \mathrm{cm}]$ which corresponds to figure 12. (b) Growth rates $\left(\omega_{i}\right)$ in dimensional form for least stable modes.
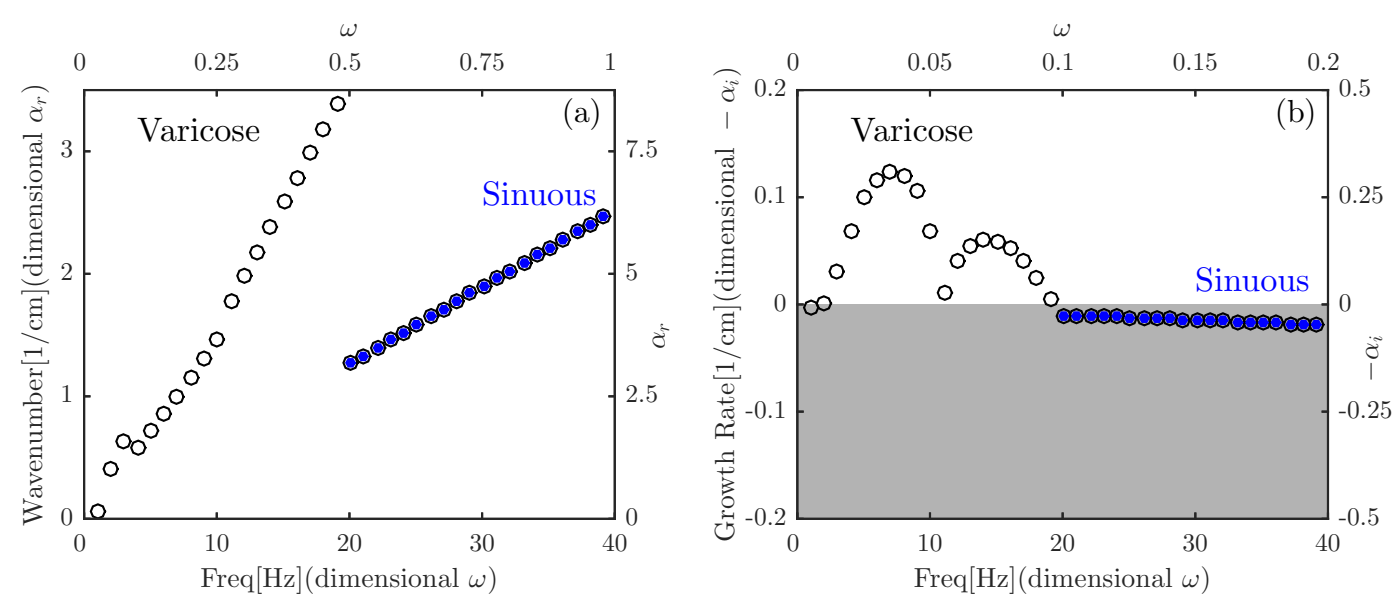

FIG. 15. (Colour online) Spatial stability analysis: least stable eigenvalues for the base-flow $U(y, z)$ in figure 5. The empty circular symbols are for the varicose modes whereas the filled circular symbols are for the sinuous modes. (a) The spatial frequency $\left(\alpha_{r}\right)$ in dimensional form corresponding to the least stable modes. (b) Growth rates $\left(-\alpha_{i}\right)$ in dimensional form for least stable modes.

equation, then substitute the disturbance form as normal modes (equation 5), and finally integrating the whole equation over one wavelength in $X$ and $Z$, and along the wallnormal direction between the two walls. The resulting equation is:

$$
\omega_{i}=\underbrace{\frac{\widehat{\mathrm{T}}_{\mathrm{y}}}{2 \mathrm{E}}}_{\omega_{i-T y}}+\underbrace{\frac{\widehat{\mathrm{T}}_{\mathrm{z}}}{2 \mathrm{E}}}_{\omega_{i-T z}}+\underbrace{\frac{-\widehat{\mathrm{D}}}{2 \mathrm{E}}}_{\omega_{i-D}},
$$


where,

$$
\left.\begin{array}{l}
\mathrm{E}=\frac{1}{2 \lambda_{z}} \int_{0}^{\lambda_{z}} \int_{-1}^{1}\left(\hat{u} \hat{u}^{*}+\hat{v} \hat{v}^{*}+\hat{w} \hat{w}^{*}\right) \mathrm{d} Y \mathrm{~d} Z, \quad \mathrm{D}=\frac{1}{2 \lambda_{z}} \int_{0}^{\lambda_{z}} \int_{-1}^{1} \frac{2}{R e}\left(\hat{\xi} \hat{\xi}^{*}+\hat{\eta} \hat{\eta}^{*}+\hat{\zeta} \hat{\zeta}^{*}\right) \mathrm{d} Y \mathrm{~d} Z \\
\widehat{\mathrm{T}}_{\mathrm{y}}=\frac{-1}{2 \lambda_{z}} \int_{0}^{\lambda_{z}} \int_{-1}^{1}\left(\hat{u} \hat{v}^{*}+\hat{u}^{*} \hat{v}\right) \frac{\partial U}{\partial y} \mathrm{~d} Y \mathrm{~d} Z, \quad \widehat{\mathrm{T}}_{\mathrm{z}}=\frac{-1}{2 \lambda_{z}} \int_{0}^{\lambda_{z}} \int_{-1}^{1}\left(\hat{u} \hat{w}^{*}+\hat{u}^{*} \hat{w}\right) \frac{\partial U}{\partial z} \mathrm{~d} Y \mathrm{~d} Z,
\end{array}\right\}
$$

and, $(\xi, \eta, \zeta)$ are the perturbation vorticity components.

\section{Spatial stability}

Similar to the temporal case, one can derive an expression for the spatial growth rates. The spatial case, however, is new and therefore we present more details in this case. The kinetic energy of spatial evolution is obtained by integrating the linearised Navier-Stokes equations (2) over one time period and in both $Y$ and $Z$ directions, over one spanwise wavelength $\lambda_{z}$ and from the top to the bottom wall. The resulting equation for the kinetic energy $e=\left(u^{2}+v^{2}+w^{2}\right) / 2$ is

$$
\frac{\partial E}{\partial x}=T_{y}+T_{z}+P-D
$$

where we have defined:

$$
\left.\begin{array}{c}
E=\frac{1}{2 T \lambda_{z}} \int_{0}^{\lambda_{z}} \int_{-1}^{1} \int_{0}^{T} e U \mathrm{~d} t \mathrm{~d} Y \mathrm{~d} Z, \\
T_{y}=\frac{1}{2 T \lambda_{z}} \int_{0}^{\lambda_{z}} \int_{-1}^{1} \int_{0}^{T}(-u v) \frac{\partial U}{\partial y} \mathrm{~d} t \mathrm{~d} Y \mathrm{~d} Z, T_{z}=\frac{1}{2 T \lambda_{z}} \int_{0}^{\lambda_{z}} \int_{-1}^{1} \int_{0}^{T}(-u w) \frac{\partial U}{\partial z} \mathrm{~d} t \mathrm{~d} Y \mathrm{~d} Z, \\
P=\frac{1}{2 T \lambda_{z}} \int_{0}^{\lambda_{z}} \int_{-1}^{1} \int_{0}^{T}(-\nabla p \cdot \boldsymbol{u}) \mathrm{d} t \mathrm{~d} Y \mathrm{~d} Z, \quad D=\frac{1}{2 T \lambda_{z}} \frac{1}{R e} \int_{0}^{1} \int_{-1}^{T}\left(-\boldsymbol{u} \cdot \nabla_{0}^{2} \boldsymbol{u}\right) \mathrm{d} t \mathrm{~d} Y \mathrm{~d} Z .
\end{array}\right\}
$$

The quantity $E$ is related to the cross-section kinetic energy in one period and $D$ is related to the viscous dissipation. The terms $T_{y}$ and $T_{z}$ are the energy production terms associated with the wallnormal and spanwise shear, respectively. The quantity $P$ is related to the work of the pressure gradient. Using the vector identity $(\boldsymbol{\nabla} \cdot(p \boldsymbol{u})=\boldsymbol{\nabla} p \cdot \boldsymbol{u}+p \boldsymbol{\nabla} \cdot \boldsymbol{u})$ and taking into account continuity, $P$ can be rewritten as $P=-\frac{\partial Q}{\partial x}$, where,

$$
Q=\frac{1}{2 T \lambda_{z}} \int_{0}^{\lambda_{z}} \int_{-1}^{1} \int_{0}^{T} p u \mathrm{~d} t \mathrm{~d} Y \mathrm{~d} Z
$$

Furthermore, defining $E_{T}=E+Q$, one can rewrite (C3) as

$$
\frac{\partial E_{T}}{\partial x}=T_{y}+T_{z}-D
$$

Now, taking the spatial normal mode expansion becomes possible. Therefore, by denoting $\left(E_{T}, D, T_{y}, T_{z}\right)=\left(\hat{E}_{T}, \hat{D}, \hat{T}_{y}, \hat{T}_{z}\right) e^{-2 \alpha_{i} x}$, the above terms may be rewritten as, 


$$
\left.\begin{array}{l}
\hat{E}_{T}=\frac{1}{2 \lambda_{z}} \int_{0}^{\lambda_{z}} \int_{-1}^{1}(\hat{e} U+\hat{q}) \mathrm{d} Y \mathrm{~d} Z, \quad \hat{D}=\frac{1}{2 \lambda_{z}} \int_{0}^{\lambda_{z}} \int_{-1}^{1} \hat{d} \mathrm{~d} Y \mathrm{~d} Z, \\
\hat{T}_{y}=\frac{1}{2 \lambda_{z}} \int_{0}^{\lambda_{z}} \int_{-1}^{1} \hat{\tau}_{u v} \frac{\partial U}{\partial y} \mathrm{~d} Y \mathrm{~d} Z, \quad \hat{T}_{z}=\frac{1}{2 \lambda_{z}} \int_{0}^{\lambda_{z}} \int_{-1}^{1} \hat{\tau}_{u w} \frac{\partial U}{\partial z} \mathrm{~d} Y \mathrm{~d} Z,
\end{array}\right\}
$$

where,

$$
\left.\begin{array}{c}
\hat{e}=\hat{u} \hat{u}^{*}+\hat{v} \hat{v}^{*}+\hat{w} \hat{w}^{*}, \quad \hat{q}=\hat{p} \hat{u}^{*}+\hat{p}^{*} \hat{u}, \\
\hat{\tau}_{u v}=-\left(\hat{u} \hat{v}^{*}+\hat{u}^{*} \hat{v}\right), \quad \hat{\tau}_{u w}=-\left(\hat{u} \hat{w}^{*}+\hat{u}^{*} \hat{w}\right) . \\
\hat{d}=-\left(\hat{u}(\hat{L} \hat{u})^{*}+\hat{u}^{*}(\hat{L} \hat{u})+\hat{v}(\hat{L} \hat{v})^{*}+\hat{v}^{*}(\hat{L} \hat{v})+\hat{w}(\hat{L} \hat{w})^{*}+\hat{w}^{*}(\hat{L} \hat{w})\right) / \operatorname{Re},
\end{array}\right\}
$$

and the operator,

$$
\hat{L}=-\alpha^{2}+\frac{\partial^{2}}{\partial y^{2}}+\frac{\partial^{2}}{\partial z^{2}}
$$

The following identity is immediately derived from eq. (C6):

$$
-\alpha_{i}=\underbrace{\frac{\hat{T}_{y}}{2 \hat{E}_{T}}}_{\alpha_{i-T y}}+\underbrace{\frac{\hat{T}_{z}}{2 \hat{E}_{T}}}_{\alpha_{i-T z}}+\underbrace{\frac{-\hat{D}}{2 \hat{E}_{T}}}_{\alpha_{i-D}}
$$

The above equation provides insight into the mechanism of instability in the spatial scenario similarly to the temporal case. It should be noted that the spatial stability requires the use of (a priori obtained) $\alpha$ and the eigenfunctions for the calculation of the growth rate components. This is unlike the temporal case, where $\omega$ is not required for the calculation of the growth rate components, rather only the a priori obtained eigenfunctions.

${ }^{1}$ S. J. Kline, W. C. Reynolds, F. A. Schraub, and P. W. Runstadler, "The structure of turbulent boundary layers," J. Fluid Mech. 30, 741-773 (1967).

${ }^{2}$ S. K. Robinson, "Coherent motions in the turbulent boundary layers," Ann. Rev. Fluid Mechanics 23, 601-639 (1991).

${ }^{3}$ R. L. Panton, Self-Sustaining Mechanisms of Wall Turbulence (Computational Mechanics Publications, Southampton, 1997).

${ }^{4}$ P. S. Klebanoff, K. D. Tidstrom, and L. M. Sargent, "The three-dimensional nature of boundary layer instability," J. Fluid Mech. 12, 1 (1962).

${ }^{5}$ T. Fric and A. Roshko, "Vortical structure in the wake of a transverse jet," J. Fluid Mech. 279, 1-47 (1994).

${ }^{6}$ R. M. Kelso, T. Lim, and A. Perry, "An experimental study of round jets in cross-flow," J. Fluid Mech. 306, 111-144 (1996).

${ }^{7} \mathrm{~K}$. Mahesh, "The interaction of jets with crossflow," Annual Review of Fluid Mechanics 45, 379-407 (2013).

${ }^{8}$ S. Megerian, J. Davitian, L. de B Alves, and A. Karagozian, "Transverse-jet shear-layer instabilities. part 1. experimental studies," J. Fluid Mech. 593, 93-129 (2007).

${ }^{9}$ S. Bagheri, P. Schlatter, P. J. Schmid, and D. S. Henningson, "Global stability of a jet in crossflow," J. Fluid Mech. 624, 33-44 (2009).

${ }^{10}$ A. R. Karagozian, "The jet in crossflowa)," Phys. Fluids 26, 101303 (2014).

${ }^{11}$ T. Cambonie and J.-L. Aider, "Transition scenario of the round jet in crossflow topology at low velocity ratios," Phys. Fluids 26, 084101 (2014).

${ }^{12} \mathrm{~A}$. Svizher and J. Cohen, "Holographic particle image velocimetry measurements of hairpin vortices in a subcritical air channel flow," Phys. Fluids 18, 014105 (2006).

${ }^{13}$ J. Philip, A. Svizher, and J. Cohen, "Scaling law for subcritical transition in plane poiseuille flow," Phys. Rev. Lett. 98, 154502 (2007).

${ }^{14}$ G. Lemoult, J.-L. Aider, and J. E. Wesfreid, "Experimental scaling law for the subcritical transition to turbulence in plane poiseuille flow," Phys. Rev. E 85, 025303 (2012). 
${ }^{15}$ M. T. Landahl, "A note on an algebraic instability of inviscid parallel shear flows," J. Fluid Mech. 98, $243(1980)$

${ }^{16}$ L. H. Gustavsson, "Energy growth of three-dimensional disturbances in plane poiseuille flow," J. Fluid Mech. 224, 241 (1991).

${ }^{17}$ M. Matsubaray and P. H. Alfredsson, "Disturbance growth in boundary layers subjected to free-stream turbulence," J. Fluid Mech. 430, 149-168 (2001).

${ }^{18}$ P. J. Schmid and D. S. Henningson, "Stability and transition in shear flows" (Springer-Verlag New York, 2001).

${ }^{19}$ W. Schoppa and F. Hussain, "Coherent structure generation in near wall turbulence," J. Fluid Mech. 453, 57-108 (2002).

${ }^{20}$ S. I. Chernyshenko and M. F. Baig, "The mechanism of streak formation in near-wall turbulence," J. Fluid Mech. 544, 99-131 (2004).

${ }^{21}$ R. J. Adrian, C. D. Meinhart, and C. D. Tomkins, "Vortex organization in the outer region of the turbulent boundary layer," J. Fluid Mech. 422, 1-54 (2000).

${ }^{22}$ A. H. Haidari and C. R. Smith, "The generation and regeneration of single hairpin vortices," J. Fluid Mech. 277, 135-162 (1994).

${ }^{23}$ J. Zhou, R. J. Adrian, S. Balachandar, and T. M. Kendall, "Mechanism for generating coherent packets of hairpin vortices in channel flow," J. Fluid Mech. 397, 353-396 (1999).

${ }^{24}$ V. Suponitsky, J. Cohen, and P. Z. Bar-Yoseph, "The generation of streaks and hairpin vortices from a localized vortex embedded in unbounded uniform shear flow," J Fluid Mech 535, 65-100 (2005).

${ }^{25} \mathrm{E}$. Malkiel, V. Levinski, and J. Cohen, "The evolution of a localized vortex disturbance in external shear flows. part 2. comparison with experiments in rotating shear flows," J Fluid Mech 379, 351-380 (1999).

${ }^{26}$ J. Cohen, V. Suponitsky, P. Z. Bar-Yoseph, A. Svizher, and J. Philip, "Localized disturbances and their relation to turbulent shear flows," AIAA Paper No. 2006-3226 (36th AIAA Fluid Dynamics Conference and Exhibit, San Francisco, California, USA, June 5-8, 2006).

${ }^{27}$ M. Skote, J. H. Haritonidis, and D. S. Henningson, "Varicose instabilities in turbulent boundary layers," Phys. Fluids 14, 2309-2323 (2002).

${ }^{28}$ M. Asai, M. Minagawa, and M. Nishioka, "The instability and breakdown of a near-wall low-speed streak," J. Fluid Mech. 455, 289-314 (2002).

${ }^{29}$ M. Karp and J. Cohen, "Tracking stages of transition in couette flow analytically," J. Fluid Mech. 748, 896-931 (2014).

${ }^{30}$ P. Schlatter, L. Brandt, H. C. de Lange, and D. S. Henningson, "On streak breakdown in bypass transition," Phys. Fluids 20, 101505 (2008)

${ }^{31}$ O. Y. Zikanov, "On the stability of pipe poiseuille flow," Phys. Fluids 8, 2923-2932 (1996).

${ }^{32}$ A. Meseguer, "Streak breakdown instability in pipe poiseuille flow," Phys. Fluids 15, 1203-1213 (2003).

${ }^{33}$ J. Philip and J. Cohen, "Formation and decay of coherent structures in pipe flow," J. Fluid Mech. 655, 258-279 (2010).

${ }^{34} \mathrm{P}$. Elofsson, M. Kawakami, and P. Alfredsson, "Experiments on the stability of streamwise streaks in plane poiseuille flow," Phys. Fluids 11, 915-930 (1999).

${ }^{35} \mathrm{~J}$. Cohen, M. Karp, and V. Mehta, "A minimal flow-elements model for the generation of packets of hairpin vortices in shear flows," J. Fluid Mech. 747, 30-43 (2014).

${ }^{36} \mathrm{~J}$. Philip, The relationship between streaks and hairpin vortices in subcritical wall bounded shear flows., Ph.D. thesis, Technion - Israel Institute of Technology (2009).

${ }^{37}$ D. S. Park and P. Huerre, "Primary and secondary instabilities of the asymptotic suction boundary layer on a curved plate," J. Fluid Mech. 283, 249-272 (1995).

${ }^{38} \mathrm{~W}$. Reynolds and A. Hussain, "The mechanics of an organized wave in turbulent shear flow. part 3. theoretical models and comparisons with experiments," J. Fluid Mech. 54, 263-288 (1972).

${ }^{39}$ M. T. Landahl, "Wave mechanics of breakdown," J. Fluid Mech. 56, 775 (1972).

${ }^{40} \mathrm{C}$. Cossu and L. Brandt, "On tollmien-schlichting-like waves in streaky boundary layers," Eur. J. Mech. B/Fluids 23, 815-833 (2004).

${ }^{41}$ J. H. Fransson, A. Talamelli, L. Brandt, and C. Cossu, "Delaying transition to turbulence by a passive mechanism," Phys. Rev. Lett. 96, 064501 (2006).

${ }^{42}$ S. C. Reddy, P. J. Schmid, J. S. Baggett, and D. S. Henningson, "On stability of streamwise streaks and transition thresholds in plane channel flows," J. Fluid Mech. 365, 269-303 (1998). 